$0014+40432$

$u C-4$

LBL-6601 C. $/$

\title{
STUDIES OF ENERGY TRANSFER PROCESSES IN \\ TRIPLET STATES USING OPTICALLY \\ DETECTED MAGNETIC RESONANCE
}

Mark Thomas Lewellyn

(Ph. D. thesis)

June 1977

Prepared for the U. S. Energy Research and

Development Administration under Contract W-7405-ENG -48

\section{For Reference}

Not to be taken from this room

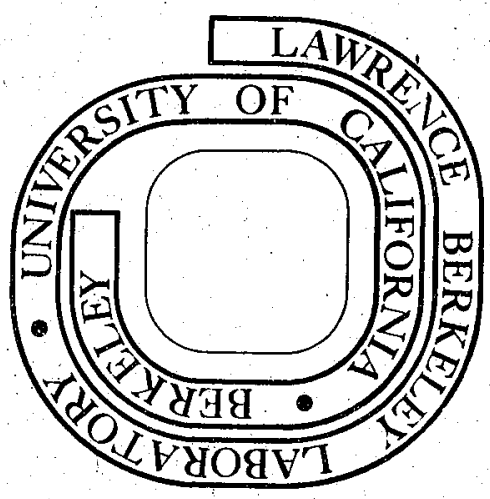


This report was prepared as an account of work sponsored by the United States Government. Neither the United States nor the United States Energy Research and Development Administration, nor any of their employees, nor any of their contractors, subcontractors, or their employees, makes any warranty, express or implied, or assumes any legal liability or responsibility for the accuracy, completeness or usefulness of any information, apparatus, product or process disclosed, or represents that its use would not infringe privately owned rights. 


\author{
STUDIES OF ENERGY TRANSFER PROCESSES IN TRIPLET STATES \\ USING OPTICALLY DETECTED MAGNETIC RESONANCE
}

Mark Thomas Lewellyn

Materials and Molecular Research Division

and Department of Chemistry

Lawrence Berkeley Laboratory

University of California

Berkeley, California 94720

June 1977

\begin{abstract}
The techniques of both continuous wave and pulsed-coherent optically detected magnetic resonance spectroscopy are used to study energy transfer processes in the lowest triplet states of two aromatic molecular crystals. of particular interest are the effects of crystal dimensionality and isotopic trap states on the efficiency of the transfer process in these systems.
\end{abstract}

The relationship between the exciton band-to-band microwave transition lineshape of a one-dimensional crystal and the properties of coherent exciton migration is developed and discussed in terms of coherent 
exciton trapping experiments on isotopically-mixed single crystals of 1,2,4,5-tetrachlorobenzene (TCB). Limitations on coherent exciton energy transfer imposed by the presence of isotopic trap inolecules are discussed in terms of trap concentration and reduced coherence times and coherence lengths. Experimental data for dilute trap and concentrated trap isotopically-mixed TCB crystals are presented which indicato that exciton-trap interactions are the prime cause of loss of exciton coherence in crystals with trap concentrations of ca. $5 \%$.

The transfer of energy between the two types of localized, isotopic trap states in TCB-mixed crystals is studied using the techniques of optically detected spin locking and temperature dependence of phosphorescence. Direct energy transfer between localized states via virtual coupling with the host states is demonstrated as well as indirect energy transfer via a host crystal exciton. The results indicate that the transfer mechanisms between trap and host states are very sensitive to the trap concentration and to the temperature of the lattice and that direct exchange is dominant at high trap concentrations.

The dimensionality of energy transfer in pyrimidine single crystals is discussed in terms of previously reported experimental data. Optical and optically detected magnetic resonance experiments on isotopicallymixed pyrimidine sing1e crystals are reported which attempt to determine the crystal dimensionality by measuring parameters from the spectra of dimeric trap molecules. In addition, evidence is shown which indicates the existence of very efficient electron-phonon coupling in excited triplet states of pyrimidine single crystals. 


\section{1}

i i i

TABLE OF CONTENTS

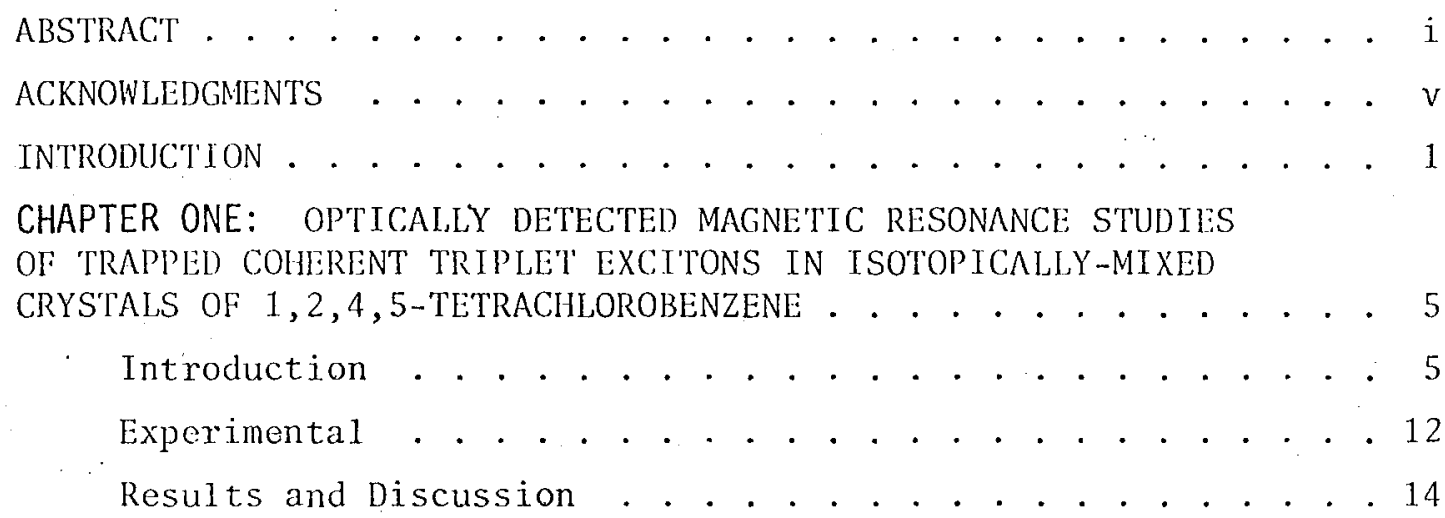

CHAPTER TWO: DIRECT AND INDTRECT ENERGY EXCHANGE BETWEEN LOCALIZED TRAP STATES . . . . . . . . . . . . . . . . 22

Introduction . . . . . . . . . . . . . . . 22

Optically Detected Spin Locking and the Kinetics of

Energy Promotion . . . . . . . . . . . . . . . . 23

Experimental . . . . . . . . . . . . . . . . 27

Results and Discussion . . . . . . . . . . . . . . 29

Temperature Dependence of Phosphorescence . . . . . 29

Dynamics of Energy Promotion: Direct and

Indirect Transfer Processes in TCB . . . . . . . . . 35

Excitation Yields and Transfer Times in

Isotopically-Mixed Crystals . . . . . . . . . . 41

Additional Considerations . . . . . . . . . . . . . 43

CHAPTER THREE: USE OF OPTICALLY DETECTED MAGNETIC RESONANCE

AS A PROBE OF THE DIMENSIONALITY OF ENERGY TRANSFER IN

PYRIMIDINE SINGLE CRYSTALS . . . . . . . . . . . . . . . . . . 46

Introduction . . . . . . . . . . . . . . . . . . 46

lixperimental . . . . . . . . . . . . . . . . . . . . . 53

Results and Discussion . . . . . . . . . . . . . 56

The Lower Excited States of the Pyrimidine Molecules . 56

Characterization of the Isotopically-Mixed

Pyrimidine Crystal System . . . . . . . . . . . 59

Optical and Optically Detected Magnetic Resonance Spectra of Isotopically-Mixed Pyrimidine Single

Crystals ................... 63

REFERENClS . . . . . . . . . . . . . . . . . . ... . . 73 

To my famizies and friends

in Minnesota and California

To see a World in a Grain of Sand And a Heaven in a Wild Flower, Hold Infinity in the palm of your hand And Eternity in an hour.

- from the Auquries if Innocence by Willian Blake 



\section{ACKNOWLEDGMENTS}

I would like to express my sincere appreciation for the scientific and financial support of Professor Charles B. Harris during the course of this work.

In addition, I wish to thank Ahmed Zewail, Bob Shelby, Ilenry Brenner, Bill Breiland, and Mike Fayer for the many things they taught me about the theoretical and experimental aspects of the spectroscopy of solids which could not be learned from texts, courses, or seminars. Acknowledgment is made of the time and care which Marc Tarrasch devoted to proof-reading and initial drafts of this thesis.

I also thank Professor D. S. Tinti for suggesting the pyrimidine-d 4 synthesis.

Finally, a special note of appreciation to my wife Jennifer, whose presence made the last two years of my graduate career much happier than they otherwise might have been.

This work was supported in part by the Materials and Molecular Research Division of the Lawrence Berkeley laboratory under the auspices of the U. S. Energy Research and Development Administration and in part by a grant from the National Science Foundation. 



\section{INTRODUCTION}

Experimental and theoretica $\ddot{1}$ interest in the properties of energy transfer in molecular solids has been concentrated principally on two general types of crystal systems. The first type can be characterized as the pure crystal case where the rigorous translational symmetry of the crystaldattice is utilized in the formulation and detection of excited states of the crystal as a whole. In this case the theories of Frenkel ${ }^{1}$ and Davydov ${ }^{2}$ can be applied to gain an understanding of the nature of energy transfer, especially as it relates to the process of coherent exciton transport. The second type involves isolated molecules as guests in a host crystal lattice. These guests vary either isotopically, chemically, or structurally from the host molecules which comprise the majority of the molecules in the lattice. If the concentration of guest molecules is low, say one percent or less, and if the excited states of the guest and host molecules are sufficiently far apart in energy, the treatment of such isolated molecules is essentially that for an oriented, single molecule coupled to a surrounding lattice. The strength of this coupling to the lattice will then determine the routes for energy transfer to and from the guest molecules.

The work in this thesis is concerned with energy transfer processes in isotopically-mixed molecular crystals. At low $\left(<4.2^{\circ} \mathrm{K}\right)$ temperatures the guest molecules in such systems can act as energy "traps" with respect to excited state energy. Trap states are very efficiently populated at low temperatures due to the effectiveness of exciton motion in the host states as a means of energy transport to such lower energy states. Thus, by observing the effects of energy transfer on guest molecules in such 
systems, it is possible to gain information on energy transfer processes in the crystal as a whole while monitoring what are essentially individual molecules in the lattice. Of particular importance is the determination of whether or not the excited state energy transfer processes are efficient enough to allow statistical models to be used in describing the relative populations of the various energy states in the solid. The experiments discussed in the following chapters deal solely with energy transfer. processes in the lowest excited triplet states of aromatic molecular crystals, although the concepts of energy transfer involved apply equally well to singlet states.

The magnitude and direction of the interactions of the individual molecules with the rest of the lattice is of prime importance in determining the nature of energy transfer in excited states. For this reason it is advantageous to work with molecular crystals where intermolecular interactions occur predominately along one of the three crystallographic axes, thus leading to efficient energy transfer in one dimension only. In aromatic molecular crystals intermolecular interactions occur predominately via the $\pi$ electrons. 3 This leads to one-dimensional bchavior in crystals where the planar aromatic rings stack in a lamellar manner with the intermolecular separation much less along one axis than the other two. 1,2,4,5-Tetrachlorobenzene (TCB) and pyrimidine are two molecules which cxhibit this type of 1attice structure. 4,5

The cxcited triplet state of a one-dimensional, pure crystal can be characterized by an energy dispersion

$$
\mathrm{E}(\mathrm{k})=2 \beta \cos (\mathrm{ka}) \text {, }
$$

assuming only nearest neighbor interactions to be important. Here $\beta$ is 


\section{0,0404333}

the magnitude of the intermolecular interaction, a is the separation between molecules along the stacking axis, and $k$ characterizes ench of the states comprising the exciton band of the crystal. B generates a group velocity for coherent exciton motion given by $V_{g}(k)=1 / \hbar \partial \mathrm{l}(\mathrm{k}) / \partial \mathrm{k}$. The coherent nature of exciton propagation depends rigorously on the translational symmetry of the lattice, and the presence of phonons and/or lattice imperfections, which destroy the translational symetry, scrves to shorten the exciton coherence time. Each $k$ state then possesses an energy width inversely proportional to $\tau(k)$, the coherence time of the exciton $k$ state. Knowing the group velocity and coherence time, it is possible to determine the coherence length,

$$
\ell(k)=\tau(k) V_{g}(k)
$$

In the limit where lattice vibrations or imperfections reduce the coherence time to a value $\tau(k) \leqslant \hbar / 4 \beta$, exciton transfer becomes incoherent, and energy transfer must be described in terms of random hops of cxcitation from molecule to molecule in the lattice.

The main portion of this thesis will deal with the effects of isotopic trap states on the efficiency of energy transfer in solids via coherent exciton motion. Chapter One is concerned with the effects of trap states on the coherence time of host excitons in isotopically-mixed crystals of TCB. In Chapter "Two the mechanisms of "communication" between excited state population in localized trap states and the delocalized states of the host exeiton band in TCB-mixed erytals will be discussod

in terms of experiments that measure some of the associated kinetic parameters. Chapter Three will deal with onergy transfer in the pyrimidine-mixed crystal system. Of principal concern will be the relationship 
between the crystal structure and the dimensionality or topology of the transfer process.

In all of these cases it is necessary to utilize experimental techniques operating on a time scale which is sensitive to energy transfer in triplet states of molecular crystals. Optically detected magnetic resonance (ODMR) is a technique which is particularly well suited for such applications. The principles and applications of this technique as applied to phosphorescent triplet states have been discussed in detail in several review articles. ${ }^{6,7}$ ODMR combines the sensitivity of visible and ultraviolet photon detection with the versatility of continuous wave and pulsed-coherent magnetic resonance spectroscopy. Due to the nondegeneracy of the triplet state spin sublevels, ODMR can be utilized in a variety of zerofield experiments to elucidate the mechanisms and pathways of energy transfer in molecular crystals. 


\section{CHAPTER ONE}

OPTICALLY DE:TECTED MAGNETIC RESONANCE STUDIIS OF TRAPIIED COIIIRLENT

TRIPLET EXCITONS IN ISOTOPICALIY-MIXEI) CRYSTALS

OF $1,2,4,5$-TETRACHIOROBENZLNE:

Introduction

The coherent properties of one-dimensional triplet excitons, which were briefly discussed in the Introduction, are summarized in Fig. 1. As can be seen, the translationally equivalent interaction $\beta$ generates a band energy dispersion which has a group velocity profile and a density of states function associated with it. In addition, at low temperatures where the bandwidth, $4 \beta$, is greater than $k T$ (where $k$ is Boltzmann's constant), triplet excitons are characterized by a distribution function $D(k)=\rho(k) \exp [-E(k) / k T]$ which weights the density of statcs function by a Boltzmann factor and determines the partitioning of cnergy between the various k-states which comprise the band.

For one-dimensional molecular crystals such as 1,1-dibromonaphthalene where $\beta$ is relatively large $\left(\sim 7.4 \mathrm{~cm}^{-1}\right), 8$ it is possible to observe emission occurring between the individual k-states of the triplet band and those of a vibrational band of the ground state. ${ }^{9}$ This transition has an associated lineshape which reflects the "doublc-humped" characteristic of the distribution function. When $\beta$ is relativoly small $\left(<1 \mathrm{~cm}^{-1}\right)$, as it is for 1,2,4,5-tetrachlorobenzene ( $T C B$ ), such eflects are very difficult to resolve in the phosphorescence spectrum.

The observation of such phenomena is important since the separation between the double maxima would give the bandwidth, $4 \beta$, and the full lineshape and linewidth would be related to the coherence time for the exciton. 
COHERENT PROPERTIES OF EXCITONS

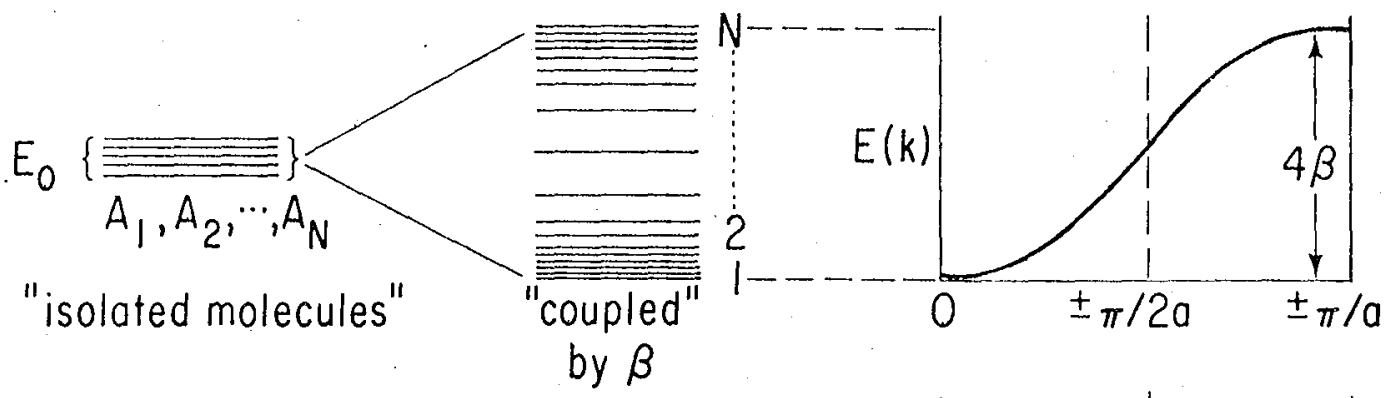

1: $\beta$ generates a rate of energy transfer between molecules: $\nu_{g}(k)$.

2. Coherence lifetime, $\tau(k)$, generates a

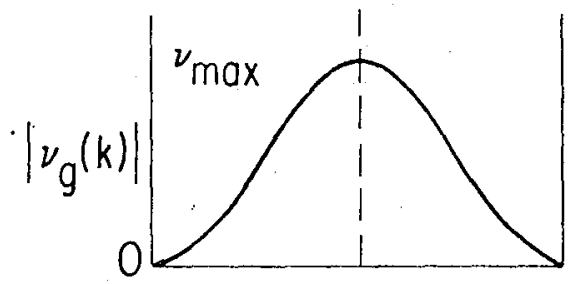
width to the state and a mean-free path for coherent energy migration

$$
\begin{aligned}
& l(k)=\nu_{g}(k) \cdot \tau(k) \\
& \tau(k)^{-1} \equiv \sum_{k^{\prime}}\left(\tau_{k k^{\prime}}\right)^{-1}=\Gamma(k)
\end{aligned}
$$

3. Distribution Function, $D(k)$, determines the partition of energy between states of different velocities

$$
D(k)_{B} \sim \rho(k) \exp [-E(k) / k T]
$$
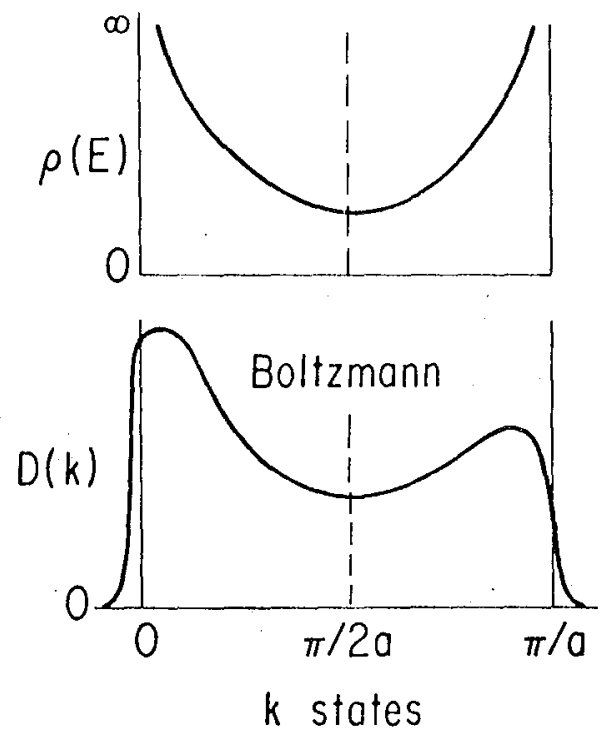

XISL. $73 .+59.4$

lig. 1. The relationship between the translationally equivalent interaction, $\beta$, the band cuergy dispersion, $l(k)$, the groul) velocity, $V_{g}(k)$, the density of states function, $\rho(l)$, and the distribution function $D(k)$, for a one-dinensional exciton. 
The latter point follows assuming that each $k-t o-k$ transition can be characterized by a lineshape function, e.g. a Lorentzian, the halfwidth of which would be related to the coherence time $\tau(k)$. The ability to determine both $\beta$ and $\tau(k)$ is critical to an understanding of what sort of effects contribute to a loss of coherence.

In their papers Francis and Harris ${ }^{10}$ showed that the observation of microwave band-to-band transitions associated with coherent triplet excitons of perprotonated TCB could be related to the bandwidth and coherence time of the exciton. Due to anisotropy in the spin-orbit coupling between the singlet manifold of states and the triplet spin sublevels, the zerofield splitting across the exciton band dispersion is not uniform; Fig. 2. This leads to a unique Larmor frequency for each $k$-state in the band-to-band transition. Each of these k-state transitions will be broadened through relaxation or energy transfer processes that lead to a loss of coherence. Therefore, the ODMR spectrum of a triplet exciton band-to-band transition should exhibit all of the features of the distribution function which characterizes the exciton at a given temperature. In this case, however, the separation between the two maxima of the double-hump lineshape will not equal 4B; rather, the separation will be related to $4 B$ by a reduction factor which takes account of the magnitude of the anisotropy in the spin-orbit coupling. Similarly, the overall width of the ODMR lineshape can be related to the coherence time. Such experiments require the preparation of high-purity, high-quality single crystals which would exhibit a maximum anount of exciton phosphorescence.

Because of this requirement for high purity, it has proved difficult 


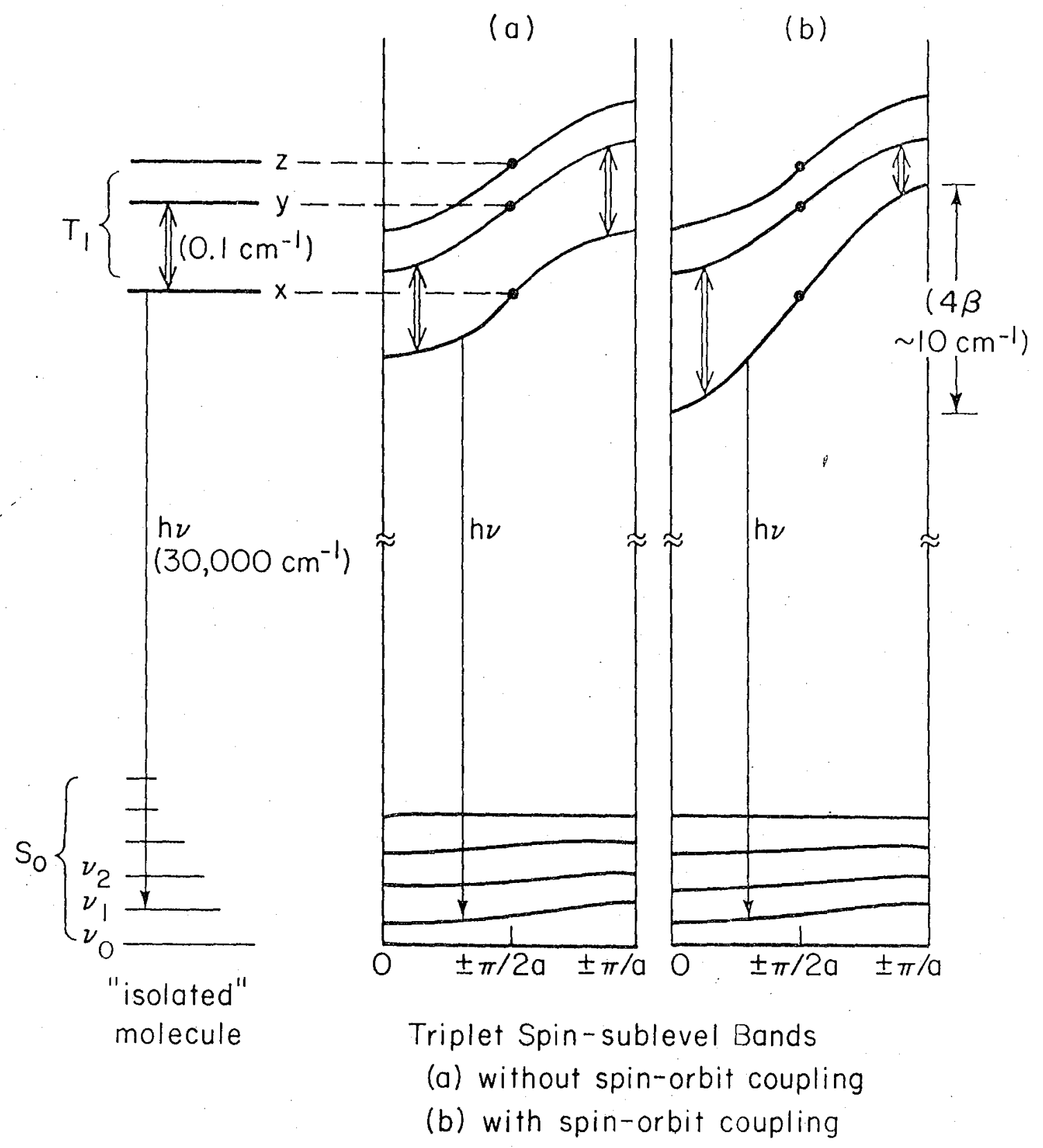

XliL $734-383$

Fig. 2. The energy dispersion of the triplet magnetic sublevel bands (a) in the absence of spin-orbit coupling, and (b) in the presence of selective spin-orbit coupling to the $x$ sublevel. Note the separations between the spin sublevels are graitly exaggcrated for illustrative purposes. 


\section{3}

$9-$

to perform similar ODMR experiments on the triplet excitons of TCB isotopically-mixed crystal systems. This inability to directly observe olnk band-to-band transitions can be circumvented by the observation of coherent exciton trapping. 11 The phenomena of coherent exciton trapping results when a microwave-induced perturbation of the exciton spin manifolds is transmitted to trapping sites via triplet exciton migration. The resulting change of trap spin alignment, then, has a direct offect on the nature of the trap ODMR spectrum. The lineshape associated with this trap-monitored exciton spin transition will depend explicitly on whether exciton-trap interaction or exciton-phonon coupling is chiefly responsible for the loss of exciton coherence.

Trap-monitored exciton spin polarization can be expressed in terms of the trap spin polarization as 11

$$
\Delta N^{t}(\omega)=K \Delta N^{c}(\omega)\left(K_{i}^{-1}+K_{j}^{-1}\right),
$$

where $\Delta N^{t}(\omega)$ is the change in trap polarization as a function of frequency, $K$ is the rate constant associated with exciton trapping, $\Delta N^{e}(\omega)$ is the change in exciton spin polarization as a function of frequency, and $k_{i}$ and $K_{j}$ are the total depletive rate constants for the trap spin sublevels. Now, for a one-dimensional Frenkel exciton

$$
\Delta N^{e}(\omega)=\rho(\omega)\{\exp [-E(\omega) / k l]\}
$$

where $\rho(\omega)$ is the density of exciton states connected by the microwave frequency $\omega$, and $E(\omega)$ is the energy dispersion of the spin manifolds.

- In the dilute trap limit, when exciton-phonon scattering is faster than trapping, the effective trapping rate constant $K \sim \bar{V}_{\mathrm{g}} \mathrm{D}_{\mathrm{T}}$, where $\mathrm{D}_{\mathrm{T}}$ is the density of trapping sites, and $\bar{V}_{g}$ is the average group velocity taken 
over the distribution of varying chain lengths within the exciton spin manifold. In this limit $\Delta N^{t}(\omega)$ depends upon the Boltzinann population distribution and the density of states $\rho(\omega)$. Consequently, the trapmonitored exciton electron spin transitions will exhibit a central band minimum associated with the minimum in $\rho(\omega)$ at $k= \pm \pi / 2$.

If, however, the principal limitation on the coherence lifetime of the triplet exciton is trapping rather than phonon scattering (concentrated trap limit), the effective trapping rate constant is taken to be proportional to the product of the group velocity of the excitons perturbed by the microwave field, $V_{g}$, and the density of trapping sites, $D_{T}$. Since $v_{g}=1 / \rho(\omega)$ for a one-dimensional exciton,

$$
\Delta N^{t}(\omega)=D_{T}\{\exp [-E(\omega) / k T]\}\left(K_{i}^{-1}+K_{j}^{-1}\right)
$$

In the concentrated trap limit, then, the change in trap spin alignment as a function of microwave frequency will depend only upon the Boltzmann distribution of the population within the exciton spin manifolds.

Studies of coherent exciton trapping using isotopically-mixed crystals are ideal. Not only are problems of guest-host solubility circumvented, but problems caused by structural defects induced in the crystal lattice by inpurity doping are also avoided. In addition, there is a capability for creating traps of varying depths with respect to the host exciton band as, for example, in crystals of TCB-d $\mathrm{d}_{2}$ where shallow traps of TCB-hd at a depth of $11 \mathrm{~cm}^{-1}$ to low energy of the TCB-d 2 band and deep traps of $\mathrm{TCB}-\mathrm{h}_{2}$ at a depth of $22 \mathrm{~cm}^{-1}$ to low energy of the band can be doped in (see Fig. 3). Another advantage of isotopically-mixed crystals arises from the fact that the ODMR resonance frequencies of the 


$$
\begin{aligned}
& 00 \% 4004339 \\
& -11-
\end{aligned}
$$

BAND AND ISOTOPIC TRAP STRUCTURE OF PERDEUTERATED $1,2,4,5$ TETRACHLOROBENZENE

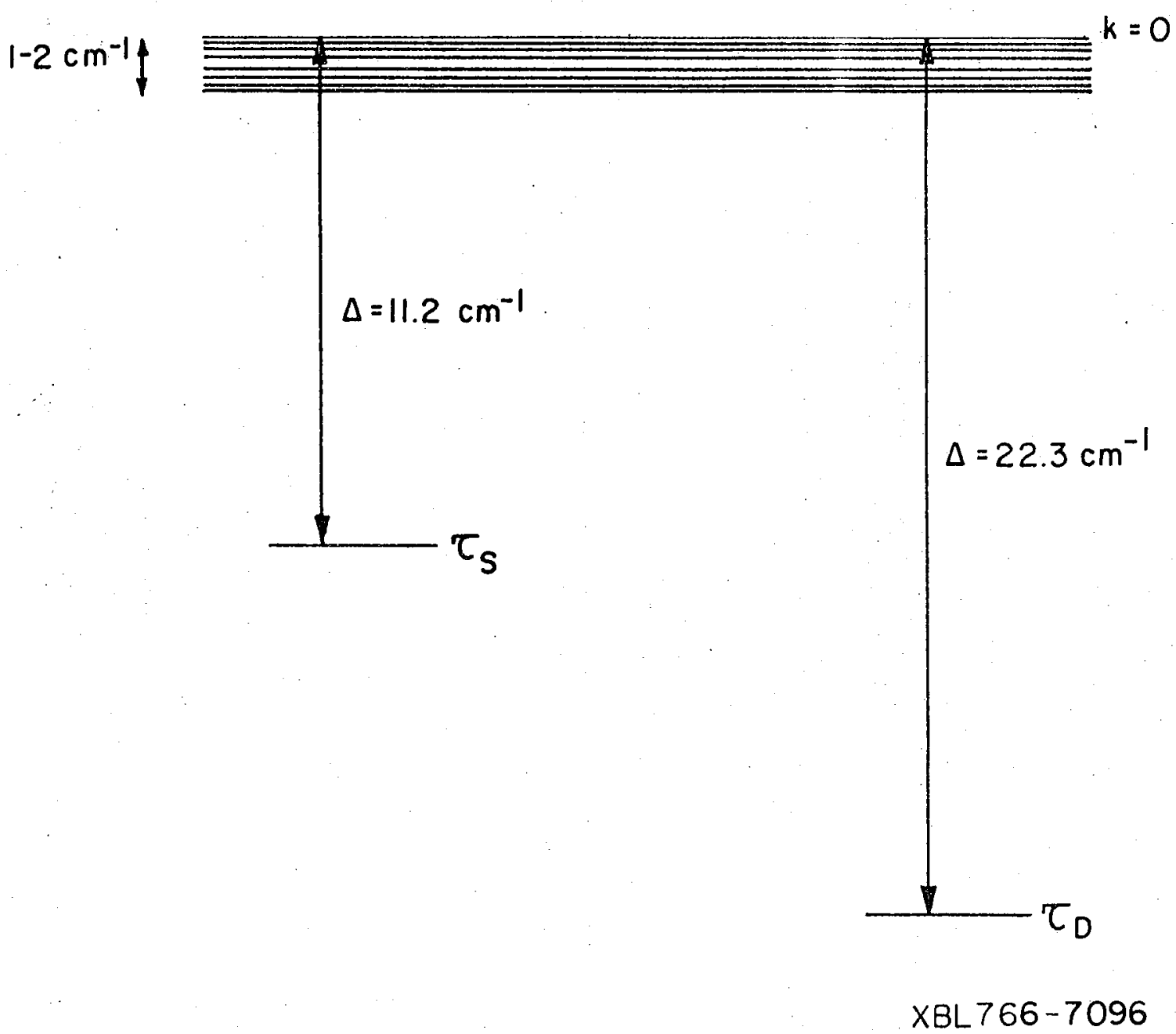

Fig. 3: The triplet energy level structure for an sotopically-mixed TCB-d $d_{2}$ crystal. $\tau_{S}$ and $\tau_{D}$ represent the shallow and decp traps respectively. The band shown is for a positive $\beta$. 
electron-spin-only transitions of the $\mathrm{TCB}-\mathrm{d}_{2}$ band and TCB-hd and $\mathrm{TCB}-\mathrm{h}_{2}$ traps should vary linearly with trap depth (assuming the exciton to have zero trap depth). This linear relationship arises from the nature of the spin-orbit perturbation which is chiefly responsible for the variation in triplet sublevel splittings with triplet state cnergy. Locating the exciton band-to-band transition then becomes a fairly easy task provided that the resonance frequencies of the two traps are known.

The observation of coherent triplet exciton trapping in isotopicallymixed molecular crystals of TCB has provided the first direct measurements of $\beta$ and coherence times for TCB-d $\mathrm{d}_{2}$ excitons as well as information on the effects of trap concentration on exciton coherence. Furthermore, these observations provide verification of the decay routes for coherently prepared trap electron spin superposition states which will be discussed in the ensuing chapter.

\section{Experimentil}

The basic experimental design for an optically detected magnetic resonance spectrometer is shown in Fig. 4. The major components of the spectrometer are: i) a microwave generator, ii) a suitably filtered, high pressure $\mathrm{Hg}$ arc lamp source for sample excitation, iii) a liquid helium cryostat containing the sample, iv) a monochromator for isolating the phosphorescence, v) a phototube; and vi) a lock-in amplifier or signal averager for processing the phototube signal. A detailed description of

- this spectrometer is given elsewhere. ${ }^{12,13}$ Two crystals were used in these experiments. The first consisted of $99.7 \% \mathrm{~d}$ TCB- $\mathrm{d}_{2}$ prepared by chlorine substitution of $99.7^{\circ} \mathrm{d}$ benzene-d $6{ }^{14}$ This crystal contained 


\section{$00+0434340$}

$-13-$

OPTICALLY DETECTED MAGNETIC RESONANCE PULSE SPECTROMETER

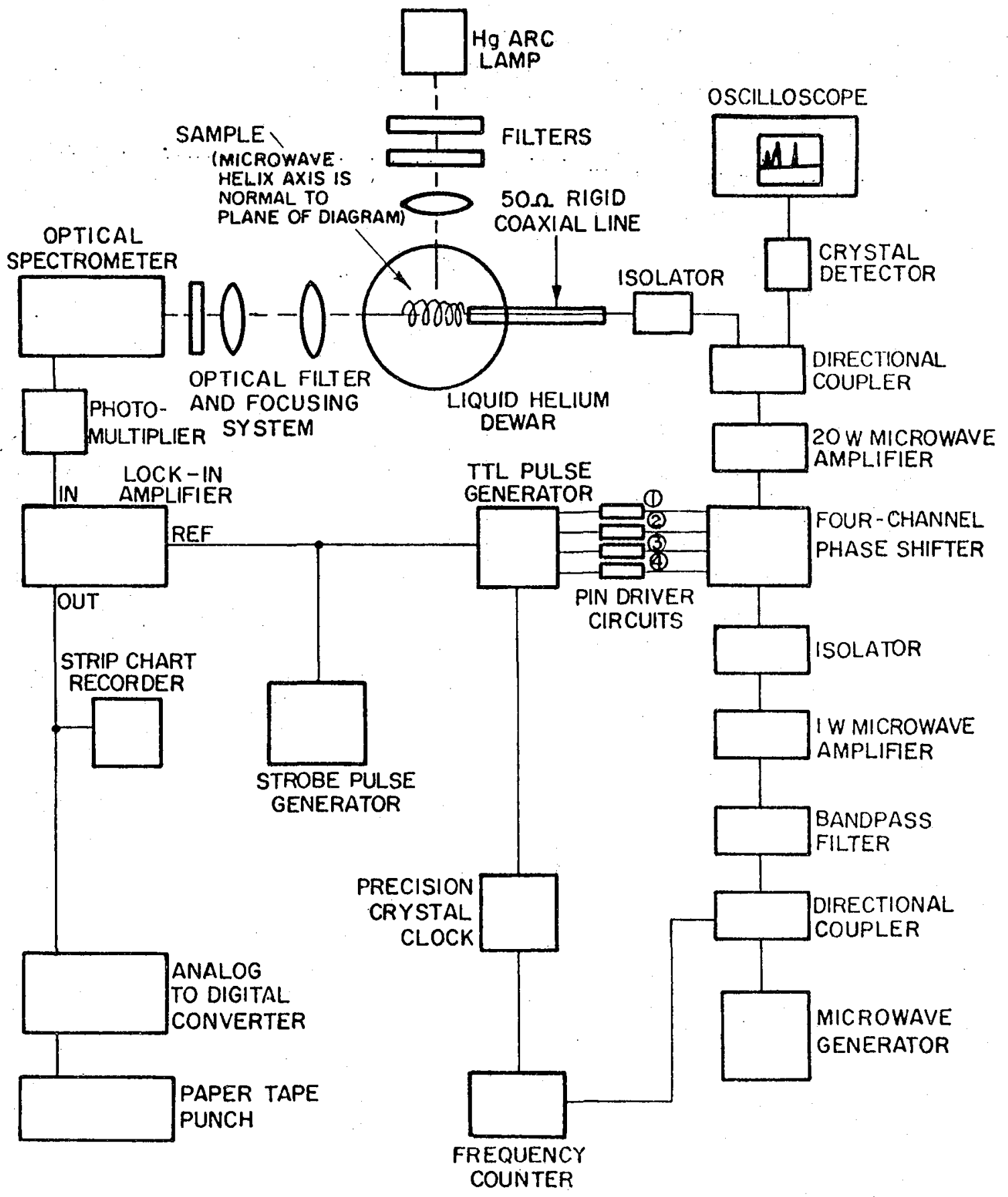

$X B L 743-5724$

Fig. 4. A block diagram for an optically detected magnetic resonance pulse spectrometer. The ITt pulse generator can be used for generating pulse sequences for coherence cxperiments such as optically detected spin locking (see Chapter Two) or for amplitude-modulating the microwave generator output in a continuous watve experiment. 
approximately $1 \%$ TCB-hd as determined by mass spectral analysis and approximately $0.006 \% \mathrm{TCB}^{-\mathrm{h}_{2}}$. The second consisted of $97.5^{\circ} \mathrm{od} \mathrm{TCB}-\mathrm{d}_{2}$ prepared by repeatedly exchanging $T C B-h_{2}$ with concentrated $\mathrm{D}_{2} \mathrm{SO}_{4}$ in $\mathrm{D}_{2} \mathrm{O}$. This crystal contained approximately $5 \%$ TCB-hd by mass spectral analysis and approximately $0.06^{\circ} \mathrm{TCB}-\mathrm{h}_{2}$. Single crystals were grown using standard Bridgman techniques. ODMR experiments were performed while monitoring emission from either the TCB-h $\mathrm{h}_{2}$ trap origin at $26668 \mathrm{~cm}^{-1}$ or from the TCB-hd trap $11 \mathrm{~cm}^{-1}$ to higher energy of the $\mathrm{TCB}_{2} \mathrm{~h}_{2}$ trip. Temperatures were varied from $1.35^{\circ}$ to $4.21^{\circ} \mathrm{K}$ by pumping on the liquid helium bath surrounding the sample. Bath temperatures were determined with a calibrated resistance thermometer placed in close proximity to the slow-wave helix containing the sample.

Results and Discussion

In Fig. 5 are shown the ODMR D-|E| transitions of the shallow (TCB-hd) and deep $\left(\mathrm{TCB}-\mathrm{h}_{2}\right)$ traps of the $99.7^{\circ} \mathrm{d}$ crystal at $1.50^{\circ} \mathrm{K}$. Evidence of coherent triplet exciton trapping can be seen in both spectra. In the spectrum of the shallow trap, the broad $(\sim 11 \mathrm{MLz})$ transition centered at about $3543 \mathrm{MHz}$ appears "positive-going" with respect to the shallow trap electron-spin-only transition at $3560 \mathrm{MHz}$, both transitions corresponding to a decrease in trap phosphorescence intensity. The slight increase in phosphorescence intensity at roughly $3578 \mathrm{Mllz}$ is duo to clectron-spin polarization associated with the deep trap which has detrapped, migrated through the exciton band, and been retrapped in a shallow trap. The spectrum of the deep trap shows similar evidence of exciton trapping. In this case electron-spin-only D-|E| transition at 
$00+400404$

D-IEI ODMR TRANSITION $99.7 \% \mathrm{~d}$ TCB- $\mathrm{d}_{2}$

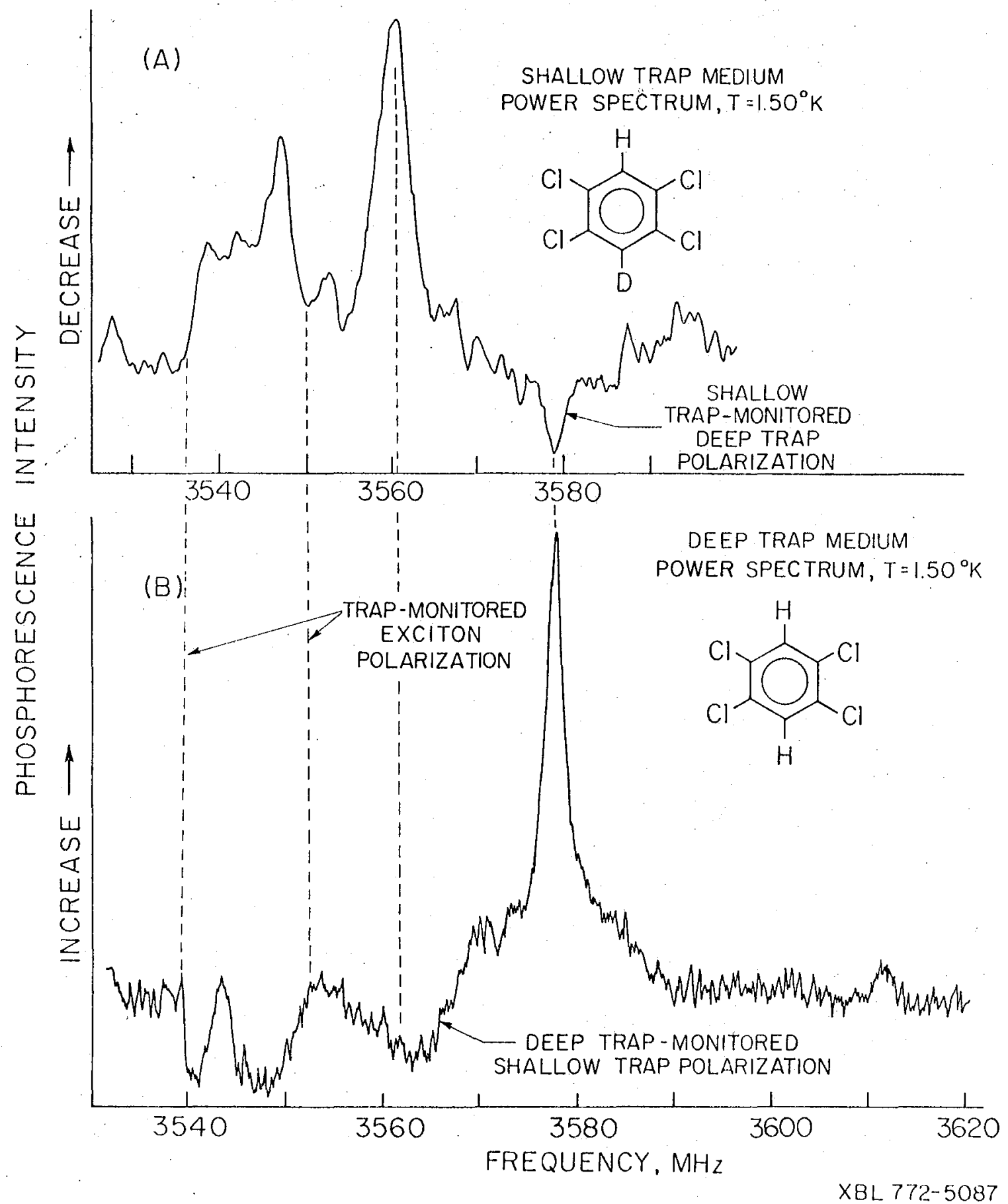

lig. 5. Trapped coherent exciton spectra for the shallow (A) and deep (B) trap D- $|E|$ transitions of a "dilute" trap $\mathrm{TCB}-\mathrm{C}_{2}$ mixed crystal. The presence of shallow trap-monitored deep trap polarization and of deep trap-monitored shallow trap polarization is also indicated. 
3577.9 MHz corresponds to an increase in phosphorescence intensity. The broad ( $11 \mathrm{MHz}$ ) "negative-going" transition centered at $3543 \mathrm{MHz}$ corresponds to the trapped $\mathrm{TCB}-\mathrm{d}_{2}$ exciton. The rather substantial dip in the transition at $3543 \mathrm{MHz}$ is due to interference from the quadrupole transitions of the TCB-h $\mathrm{h}_{2}$ trap. The additional broad, negative-going transition with a maximum at $3563 \mathrm{MHz}$ is due to shallow trap polarization that has been trapped in the deep trap. At higher temperatures $\left(>1.60^{\circ} \mathrm{K}\right)$ this transition narrows and becomes positive-going. No explanation can be offered at this time as to why the broadening at lower temperatures occurs.

The 1 inewidth and intensity of the exciton transition was insensitive to the application of a homogeneous, but arbitrarily oriented, magnetic field of 1-20 G while the electron-spin-only transition of the trap showed some broadening. At higher fields $(20-100 \mathrm{G})$ the electron-spinonly trap transition showed continued broadening which resulted in a masking of the exciton band-to-band transition. This lack of broadening of the trapped exciton resonance is consistent with motional averaging of the nuclear spin states in exciton electron spin transitions.

An expanded scale spectrum of the D- $|E|$ exciton transition trapped in the shallow trap is given in Fig. 6. This exhibits the characteristic "double-hump" appearance discussed previously. The width at half height is $11 \mathrm{MHz}$, and the two maxima are separated by $8.5 \mathrm{Mllz}$ which exactly match the results of measurements of the $\mathrm{D}-|\mathrm{E}|$ band-to-band transition in $\mathrm{TCB}-\mathrm{h}_{2}$ crystals. $^{10}$ Neither the $D+|E|$ nor the $2|\mathrm{E}|$ trapped exciton transitions of TCB-d ${ }_{2}$ were observed. In addition, the intensity of the D-|E| trapped exciton transition was extremely sensitive to temperature. The intensity 


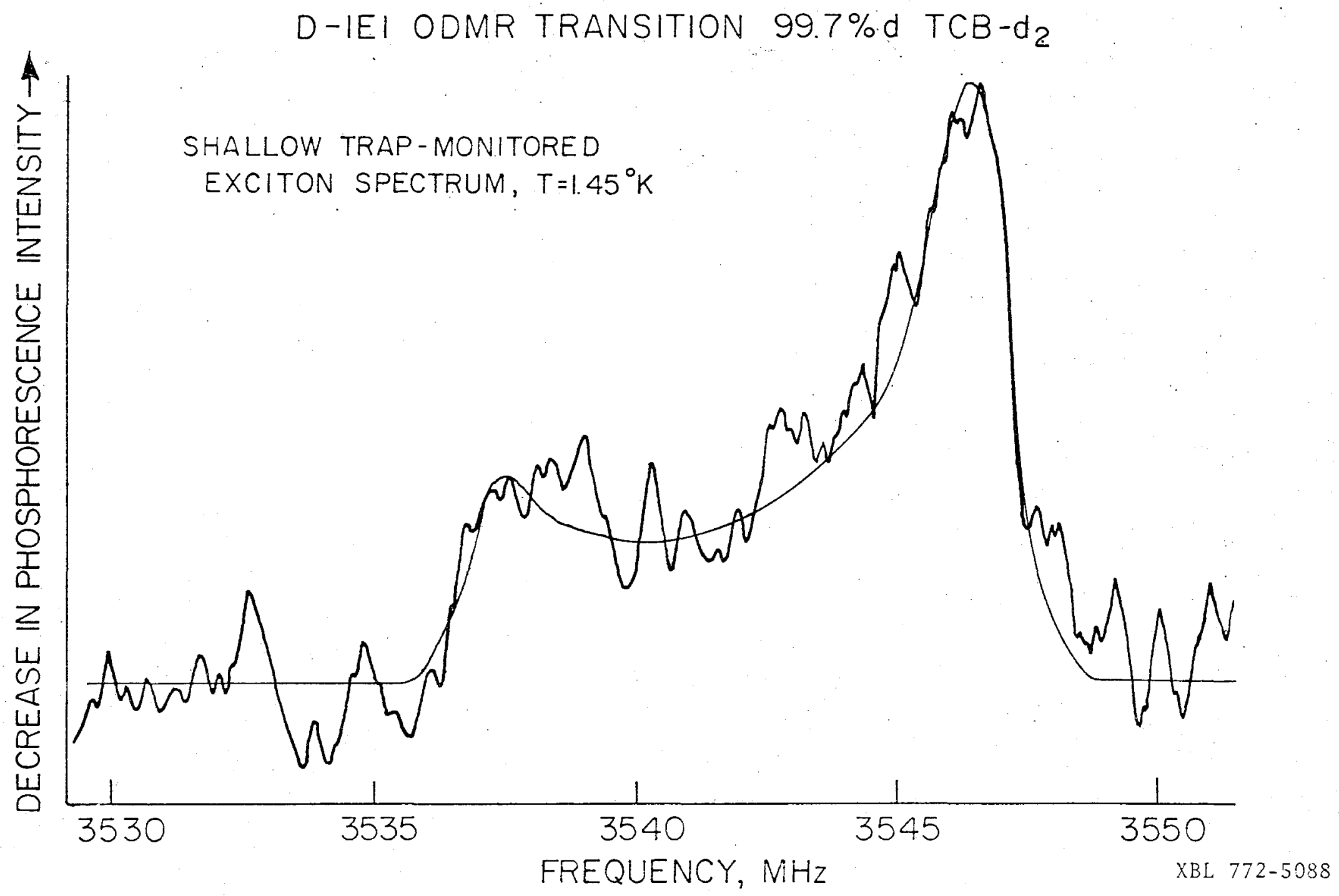

Fig. 6. An expanded scale spectrum of the shallow trap-monitored exciton D-|E| transition of the $99.7 \mathrm{~d} \mathrm{dCB}_{2} \mathrm{~d}_{2}$ crystal. The superimposed curve represents the best theoretical fit. 
decreased by roughly a factor of two from 1.40 to $1.60^{\circ} \mathrm{K}$, and the transition was unobservable above $1.70^{\circ} \mathrm{K}$.

Using the theories of Francis and Harris ${ }^{10}$ and Harris and Fayer ${ }^{16}$ which develop the relationship between the energy dispersion of the exciton band and the band-to-band microwave transitions, it is possible to fit. the ODMR data to determine the exciton bandwidth and to determine a minimum coherence time associated with the exciton. Specifically, the effect of selective spin-orbit coupling introduces an exciton wave vector (k) dependence into the microwave band-to-band transition, as mentioned previously. The microwave transition frequency in the k-domain, $\triangle E(k)$, is directly related to the band dispersion, $2 \beta \cos (k a)$, of the triplet state:

$$
[\Delta E(k)-\Delta E] f^{-1}=2 B \cos (k a)
$$

where $\Delta \mathrm{E}$ is the microwave frequency in the absence of selective spinorbit coupling, and $f$ is the reduction factor. By assuming that the transition associated with the Larmor frequency of each $k$-state is broadened by a transverse relaxation time which includes exciton-phonon scattering, a minimum coherence time can be associated with the width of a band-toband transition comprised of a given number of k-states.

The best theoretical fit to the data was achieved for a band comprised of 100 k-states, each associated with a Gaussian-broadened transition. A Gaussian broadening factor was chosen due to the randomness of chain lengths expected in the sample. The fit, which is shown in Fig. 6, gave a triplet bandwidth, $4 \beta$, of $1.20 \mathrm{~cm}^{-1}$ and a minimum coherence time of $\sim 5 \times 10^{-7} \mathrm{~s}$. This is in good agreement with the bandwidth of $1.3 \mathrm{~cm}^{-1}$ and minimum coherence time of $\sim 9 \times 10^{-7}$ s obtained by fitting the $D+|E|$ band- 
to-band transition of $\mathrm{TCB}_{2} \mathrm{~h}_{2} \cdot{ }^{10}$ These results are also consistent with the dimer parameters of TCB-h $_{2}$ reported by Zewail and llarris ${ }^{17,18}$ and also with the recent results of Dlott and Fayer. ${ }^{19}$

Exciton trapping was also observed in crystals of a higher trap concentration. The ODMR spectrum of the shallow trap of a $97.5 \% \mathrm{~d}$ TCB-d 2 crystal is shown in Fig. $7(A)$. As can be seen, the exciton transition is observable but is much weaker in intensity than in the $99.7 \%$ d crystal. An expanded scale spectrum of the same transition is shown in Fig. $7(B)$. Exciton trapping was not observed on the deep trap ODMR spectrum of the 97.5\% crysta1. Although the signal-to-noise ratio is poor, it is interesting to note that the central band minimum seems to have been removed. The superimposed theoretical fit was obtained using the same parameters for the bandwidth and broadening function as for the $99.7 \%$ crystal with the exception that the band states were not weighted by a density of states function characteristic of a one-dimensional band. For the $97.5^{\circ}$ crystal, then, trapping has become the dominant limitation to coherent migration; whereas for the dilute trap $99.7 \% \mathrm{~d}$ crystal exciton-phonon scattering is more effective.

Since the trap-state-to-band-state concentration ratio for the $99.7^{\circ} \mathrm{d}$ crystal is about $1 / 100$ as compared with that for the $97.5^{\circ} \mathrm{d}$ crystal of about $1 / 20$, the results of the coherent exciton trapping experiments on these two systems would suggest a minimum coherence $1 \mathrm{ength}$, $\ell(k)$, of at. least $20-100$ lattice sites. $\Lambda$ coherence length of 100 lattice sites corresponds to a coherence time of $\sim 10^{-9} \mathrm{~s}$ for a one-dimensional exciton with $4 \beta=1.20 \mathrm{~cm}^{-1}$. The coherence time obtained from the fit of the trapped exciton spectrum associated with the $99.7 \% \mathrm{~d}$ crystal was $\sim 5 \times 10^{-7} \mathrm{~s}$ 
D-IEI ODMR TRANSITION $97.5 \% \mathrm{~d}$ TCB-d

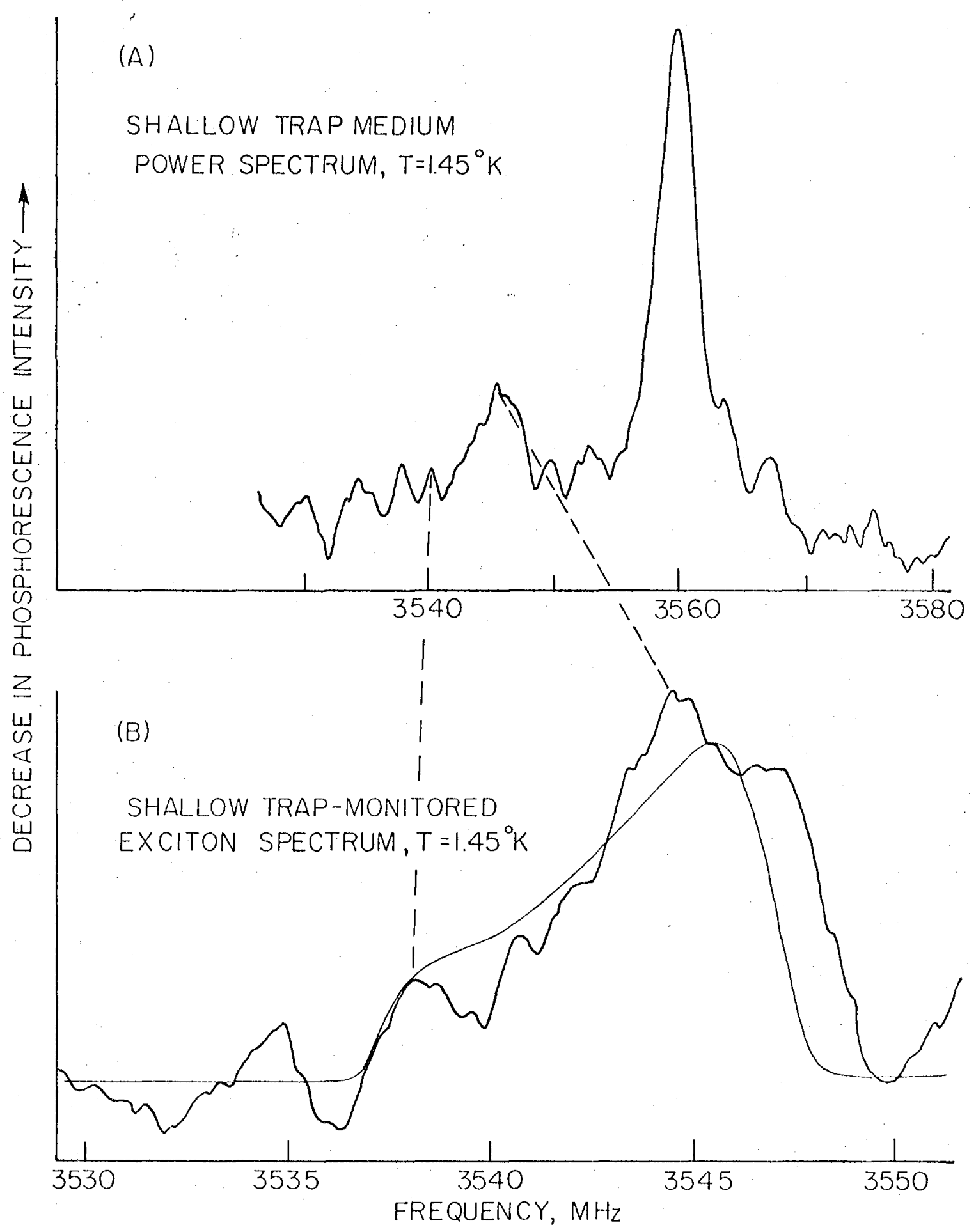

XBL 772-5089

Fig. 7. (A) The shallow trap-monitored D-|E| trapped exciton transition for the concentrated trap $T C B-d_{2}$ mixed crystal. (B) Shows the expanded scalc exciton spectrum with the superimposed theoretical fit. 


\section{$00+940+34$}

indicating a coherence length greater than 100 lattice sites and confirming that for the dilute trap case the main limitation on exciton coherence is exciton-phonon scattering. This coherence length compares favorably to that for pure $\mathrm{TCB}-\mathrm{h}_{2}$ crystals of $\sim 186$ lattice sites as determined by laser excitation experiments. 20 In the case of $T C B-h_{2}$ pure crystals; of course, the trap-state-to-band-state ratio is much smaller, the traps being due to $\mathrm{TCB}-\mathrm{h}_{2}$ molecules with orientational differences.

Several other interesting conclusions can be drawn from this data apart from the value for the bandwidth and the coherence time. By establishing the resonance frequency of the TCB- $\mathrm{d}_{2}$ exciton band-to-band transition as $3543 \mathrm{MHz}$ as compared with the $3577.9 \mathrm{MHz}$ frequency of the deep trap clectron-spin-only transition, it has been shown that detrapping processes occurring from coherently prepared superpositions of trap electron spin states ${ }^{13}$ to the band will lead to a definite loss of spin coherence due to the $35 \mathrm{MHz}$ difference in resonance frequencies. This fact will be most important for the experiments discussed in the next chapter. It is also interesting to note that by plotting trap depth versus resonance frequency for the deep and shallow traps and the exciton band (assuming the band trap depth to be zero), a linear relationship results. This nicely exhibits the effects of spin-orbit coupling on the resonance frequency of the various sites in this mixed crystal system. 


\section{CHAPTER TWO \\ DIRECT AND INDIRECT ENERGY EXCHANGE BETWEEN LOCALIZED TRAP STATES}

\section{Introduction}

It was noted in Chapter One that a mechanism for communication between the shallow and deep trap sites in isotopically-mixed TCB crystals exists. This was evident from the observation of features characteristic of the decp trap spin polarization in the shallow trap ODMR spectrum and vice versa. Both optical and magnetic resonance studies have shown that. excitations are transferred among different trap sites by at least two distinct processes. The first involves the direct ${ }^{21}$ exchange of energy between trap sites via a virtual coupling with the host states $^{22}$ while the second is an indirect ${ }^{23}$ pathway involving a decay into the delocalized host states (exciton band), migration in the band, and retrapping. By studying these processes, it is possible to obtain information not only on the resonance interactions between trap sites, but also on the nature of interactions between the trap and host states. In addition, one can extract parameters detailing the exciton band structure of the host crystal. In earlier experiments ${ }^{23}$ the relationship between the trap depth $(\Delta$, below the exciton band), trap concentration, and the relative proportion of excited state population channeled into various types of traps was obtained by investigating the phosphorescence spectra from various traps. Such measurements, however, were not capable of giving detailed information on the nature of the transfer process. This requires the use of additional techniques. 24,25 


\section{$00 \times 4304345$}

- This chapter will be concerned with the application of the techniques of temperature dependence of phosphorescence and optically detected spin locking (ODSL) 24,13 in an examination of the different routes of energy transfer. Specifically, both direct and indirect transfer between traps and the host exciton band in excited triplet states of TCB-d 2 , containing two isotopic traps below the band, will be elucidated and compared to theoretical expectations.

Optically Detected Spin Locking and the Kinetics of Energy Promotion

The essential features of the spin-locking experiment can be viewed using a geometrical representation ${ }^{26}$ for a two level system. The two level model is appropriate since at liquid helium temperatures the three triplet spin sublevels are essentially isolated from each other, and only those levels being connected by the microwaves need be considered. In the geometrical picture the time evolution of the ensemble of excited triplet states is represented as a "pseudomagnetization" whose orientation in the geometric frame can be changed by applied r.f. pulses and fields in exactly the same manner as the real magnetization in a conventional NMR experiment. $^{13}$ Initially the pseudomagnetization is aligned along the $z$ axis in the interaction representation and corresponds to the population difference between the two zeroficld spin sublevels involved. It should be noted that usually only changes in the population differences alter the phosphorescence intensity; hence the $z$ component of the pscudomagneti-. zation becomes the only observable one in an optically detected experiment. ${ }^{17}$

Spin locking 28,29 is accomplished by applying a $\pi / 2$ pulse to rotate the pseudomagnetization into the $x-y$ plane of the frame, followed by the 
application of a strong "spin-locking" $H_{1}$ field along the pseudomagnetization to prevent a loss of phase coherence. This results in a coherent triplet spin state that is destroyed whenever a fluctuation of the zerofield transition frequency (Larmor frequency) occurs that is large relative to the applied field, $\mathrm{\gamma H}_{1} \cdot{ }^{25}$ The loss of spin coherence, in this case the decay of the spin-locked pseudomagnetization, is monitored optically by applying another $\pi / 2$ "probe" pulse 30 with appropriate phase to rotate the spin-locked component back to the z-axis. This produces a change in the phosphorescence intensity proportional to the remaining coherence in the spin ensemble.

Several features of the spin-locking experiment make it particularly useful as a means for investigating energy migration. First of all, population intersystem crossing into the triplet ensemble during the course of the experiment enters the geometric frame along the $z$-axis and for "on-resonance" experiments is driven in a plane perpendicular to the spin-locked component. 23 In this manner incoming population makes no contribution to the change in phosphorescence intensity when the probe pulse is applied. More importantly, the spin-locked component is quite sensitive to energy transfer processes since most of them involve significant changes in the Larmor frequency. As pointed out in the previous chapter, energy promotion to different trap species or the host exciton band will result in a loss of spin coherence because of a large frequency fluctuation the spin-locked ensemble encounters.

Three principal processes change the total lifetime of the spinlocked pscudomagnetization, $\mathrm{T}_{1 \rho}$ ' in a one-dimensional system. These are: 


\section{$00+4=0043.9$}

i) the promotion of population from the spin-locked state to different trap states or the exciton band;

ii) processes leading to radiative and non-radiative decay of the triplet state to ground state;

iii), magnetic relaxation along the spin-locking field. Consideration of these processes leads to the following kinetic equation for the time evolution of the spin-locked pseudomagnetization, $M(t)$ :

$$
M(t)=M_{0} \exp \left(-k^{T} 1 \rho t\right)=M_{0} \exp \left[-\left(k^{p}+k^{L}+k^{T} 1 \rho m\right) t\right]
$$

where $K^{\mathrm{P}}, \mathrm{K}^{\mathrm{L}}$, and $\mathrm{K}^{\mathrm{T}} 1 \rho \mathrm{m}$ are the rate constants associated with the loss of the spin-locked signal owing to processes i), ii), and iii), respectively, and $K^{T} 1 \rho$ is the total decay rate constant. Additional decay terms must be considered if translationally inequivalent exchange becomes important. ${ }^{14}$

The results of spin-locking experiments, when combined with independent measurements of $K^{\mathrm{L}}$ and $\mathrm{K}^{\mathrm{T}} 1 \rho \mathrm{m}$, will yield $\mathrm{K}^{\mathrm{P}}$, the rate constant for promotion processes between different trap states and between trap states and the host exciton band. The temperature dependence of $K^{p}$, and hence of the promotion process, can then be used to detail the nature of the transfer mechanism (direct or indirect) as well as to amplify the qualitative results obtained from the temperature dependence of phosphorescence studies.

The various types of triplet state energy transfor in isotopicallymixed TCB $\mathrm{d}_{2}$ crystals are summarized in Fig. 8. The trap depths of the shallow (hd) and deep $\left(\mathrm{h}_{2}\right)$ states are $11 \mathrm{~cm}^{-1}$ and $22 \mathrm{~cm}^{-1}$, respectively, below the TCB-d 2 band; hence, phonon-assisted promotion of population 


\section{BAND-TRAP AND TRAP-TRAP INTERACTIONS IN TCB MIXED CRYSTAL}

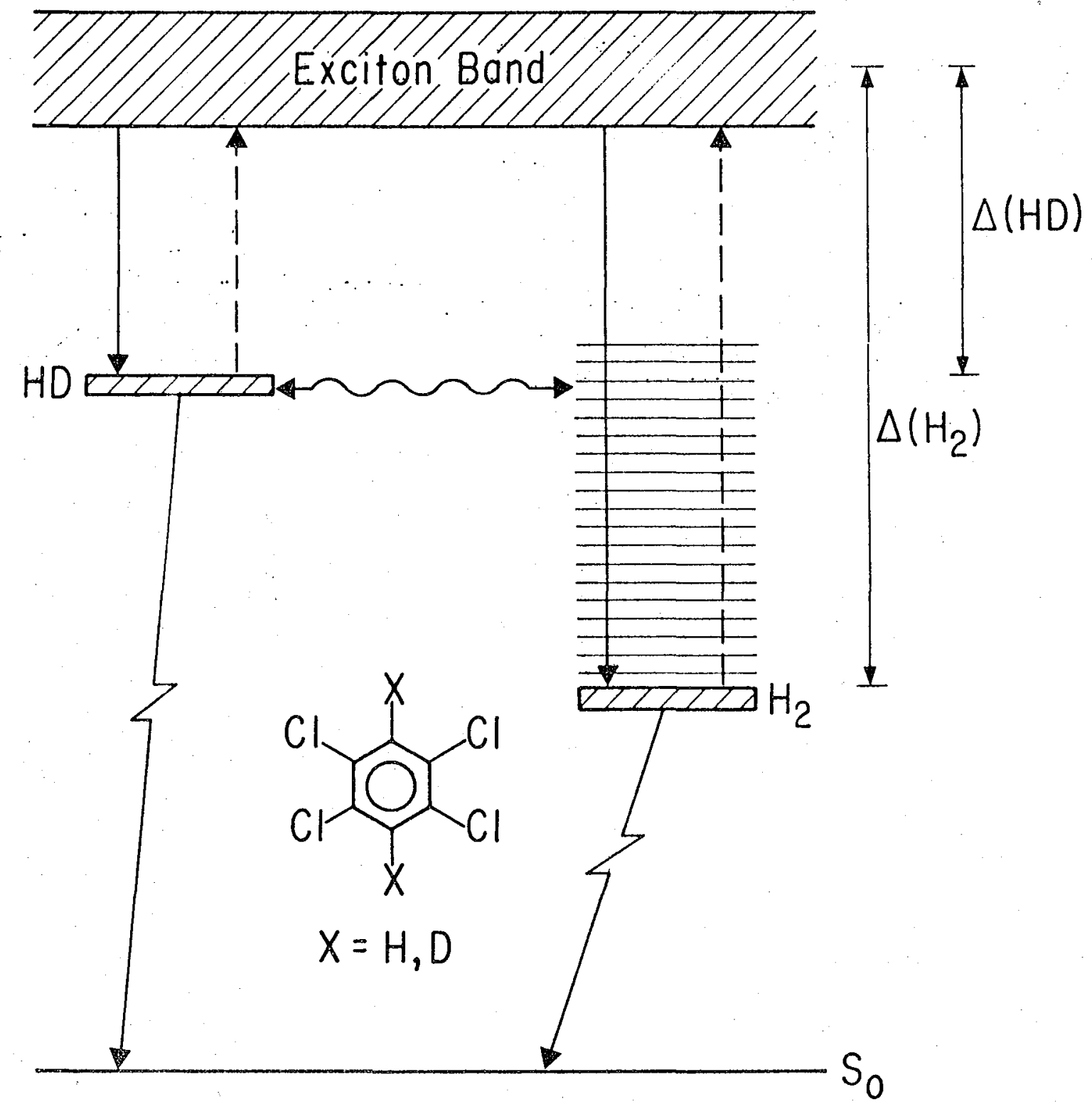

X131. 755.13978

Fig. 8. A schematic for the structure of the excited triplet state of isotopically-mixed TCB-d 2 crystals: $\Delta$ is the trap depth, and $\mathrm{H}_{2}$ or $\mathrm{Hl}$ refer to the different isotopic species. The solid arrows represent emission to the ground state or trapping in trap sites; emission of the exciton to the ground state is not shown. The dotted arrows represent detrapping processes to the band, and the wiggly double-arrowed line represents the communication between traps via a direct mechanism. 
out of the decp trap should reveal the relative importance of detrapping processes between the deep trap and the band and the deep trap and the shallow trap and their dependence on deep trap concentration and temperature.

\section{Experimental}

Two isotopically-mixed TCB samples were prepared, each containing different proportions of $\mathrm{TCB}-\mathrm{h}_{2}$. The first was prepared from $97.5 \%$ deuterated, zone-refined TCB. It contained $95.31 \% \mathrm{TCB}-\mathrm{d}_{2}, 4.63 \% \mathrm{TCB}-\mathrm{hd}$, and $0.06 \% \mathrm{TCB}-\mathrm{h}_{2}$. The second sample was prepared by adding a given amount of zone-refined TCB-h $\mathrm{h}_{2}$ to the $97.5 \% \mathrm{~d} \mathrm{TCB}-\mathrm{d}_{2}$ such that the final sample contained approximately $5 \% \mathrm{TCB}-\mathrm{h}_{2}$ on a weight-for-weight basis (more precisely, 90.5\% TCB- $\mathrm{d}_{2}, 4.6 \% \mathrm{TCB}-\mathrm{hd}$, and $\left.4.8 \% \mathrm{TCB}-\mathrm{h}_{2}\right)$. These samples (hereafter referred to as the $0.06 \%$ and $5 \%$ samples) were analyzed by either mass spectral analysis or by proton MMR. Single crystals were grown from the melt by standard Bridgman techniques. Both crystals were amealed for one week at a temperature $2.4^{\circ} \mathrm{C}$ below the melting point. The quality of each crystal was checked by its ability to give exciton phosphorescence. The exciton emission was seen in neither the $0.06 \%$ nor the $5 \%$ crystal below the lambda point of helium, although strong cmission from the exciton origin was observed at $4.2^{\circ} \mathrm{K}$ in the $0.06^{\circ}$ sample.

The experimental arrangement for optically detected magnetic resonance was discussed briefly in Chapter One. Microwave pulses of appropriate phases for the ODSL experiment were obtaincl as follows. The microwave output of a Hewlett-Packard $8690 \mathrm{~B}$ sweeper was divided into two separate channels by means of an Anaren Corp. $90^{\circ}$ hybrid coupler. 
Phases were set by adjusting the relative path lengths in the two channels by means of two $10 \mathrm{~cm}$ General Radio sliding coaxial lines. Two HewlettPackard 33124A PIN diodes were inserted into each channel to switch the microwaves. Timing of the pulse sequences was controlled by TTL circuits. The channels were recombined by means of a $180^{\circ}$ hybrid coupler, the resultant signal being fed through a band-pass filter to a Varian 615M 20 w traveling wavetube amplifier before delivery to a slow-wave helix surrounding the sample in a liquid helium cryostat. The temperature of the bath could be varied between $1.4^{\circ}$ and $4.2^{\circ} \mathrm{K}$ by pumping on the helium and was measured by monitoring the pressure of gaseous helium with an NRC Equipment Corp. model 530 Alphatron vacuum gauge. Temperature was stabilized by employing a pressure sensor capable of altering the pumping speed. $^{31}$ For all measurements at any given temperature, the sample was allowed to equilibrate with the bath for at least five minutes.

The experiments were performed as follows: The sample was illuminated by an Osram HBO $100 \mathrm{~W}$ mercury arc lamp filtered by a $3100 \AA$ interference filter. Phosphorescence from the $\mathrm{TCB}-\mathrm{h}_{2}$ trap origin was monitored, and the D- $|\mathrm{E}|$ transition at $3577.9 \mathrm{MHz}$ was pumped. Pulse lengths were determined by rotary precession experiments; ${ }^{32} \pi / 2$ pulses were typically $50-100 \mathrm{~ns}$. Phases were set by maximizing the initial spin-locking signal. The microwave experiments were repeated at a rate of $3-6 \mathrm{~Hz}$ and the signals time-averaged with a Northern NS-575 signal averager. 


\section{$00+4+3+448$}

\section{RESULTS AND DISCUSSION}

Temperature Dependence of Phosphorescence

Temperature dependence of phosphorescence studies on the $0.06 \%$ crystal revealed a marked dependence on temperature of the emission intensity for both the shallow (hd) and deep $\left(h_{2}\right)$ triaps. A phosphorescence spectrum of these two traps in the $0.06 \%$ crystal at it fixed temperature is shown in Fig. 9, and the corresponding temperature dependence of the emission spectra for the temperature region between $1.4^{\circ}$ and $2.1^{\circ} \mathrm{K}$ is shown in Fig. 10. As can be seen, the deep trap emission increases while the shallow trap emission decreases over the range. At the same time, however, the total phosphorescence intensity from the origin remains constant within $\sim 10 \%$. This result strongly suggests that excited state population is preferentially transferred from the shallow to the deep trap as the temperature is increased. It is clear that at these temperatures the communication between the two types of traps is not governed by Boltzmann statistics. If one plots the natural $\log$ of the emission intensity ratio between the shallow and deep traps, $I_{S} / I_{D}$, versus the reciprocal of the absolute temperature, one obtains a straight line plot, the slope of which is reversed in sign to that expected if Boltzmann statistics were the controlling factor. What the temperature dependence of these results does show, however, is that there is an energy "gap" of $8 \pm 3 \mathrm{~cm}^{-1}$ between the shallow trap to the band. This suggests that the transfer of excited state population from the shallow to the deep trap in the $0.06 \%$ crystal is occurring indirectly via the host crystal band states. $\wedge \operatorname{model}^{23}$ for this transfor process in the riplet state has been proposed which entails three basic steps: 


\section{Phosphorescence Origin}

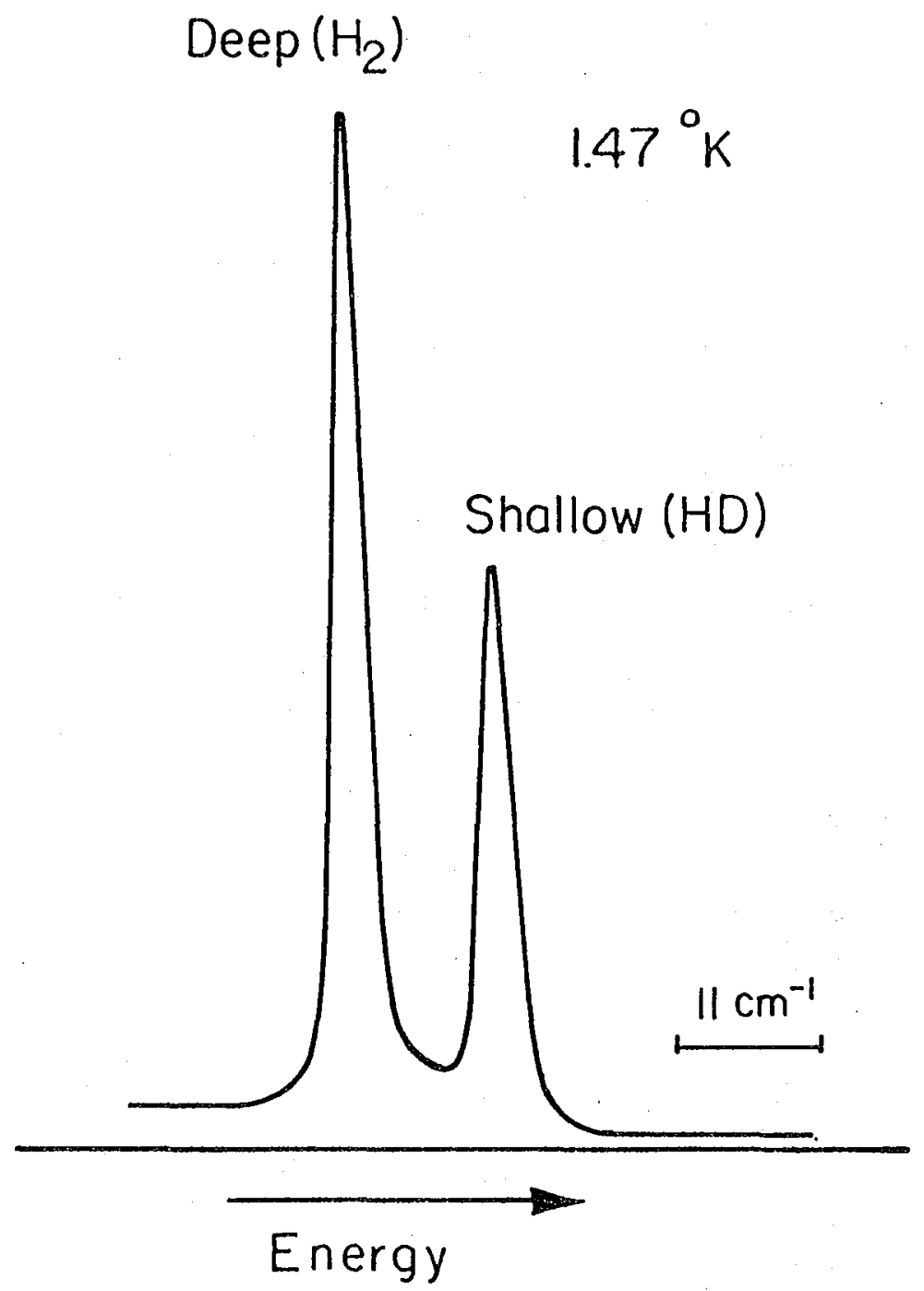

XBL $755-1399$

lig. 9. Moderate resolution phosphorescence spectrum of the pure electronic origin in isotopically-mixed TCB $(0.06 \%)$ crystals at $1.47^{\circ} \mathrm{K}$; the exciton origin appears to high energy of the IID emission at temperatures around $4.2^{\circ} \mathrm{K}$, and $\mathrm{H}_{2}$ emission is located at $26668 \mathrm{~cm}^{-1}$. 
004040439

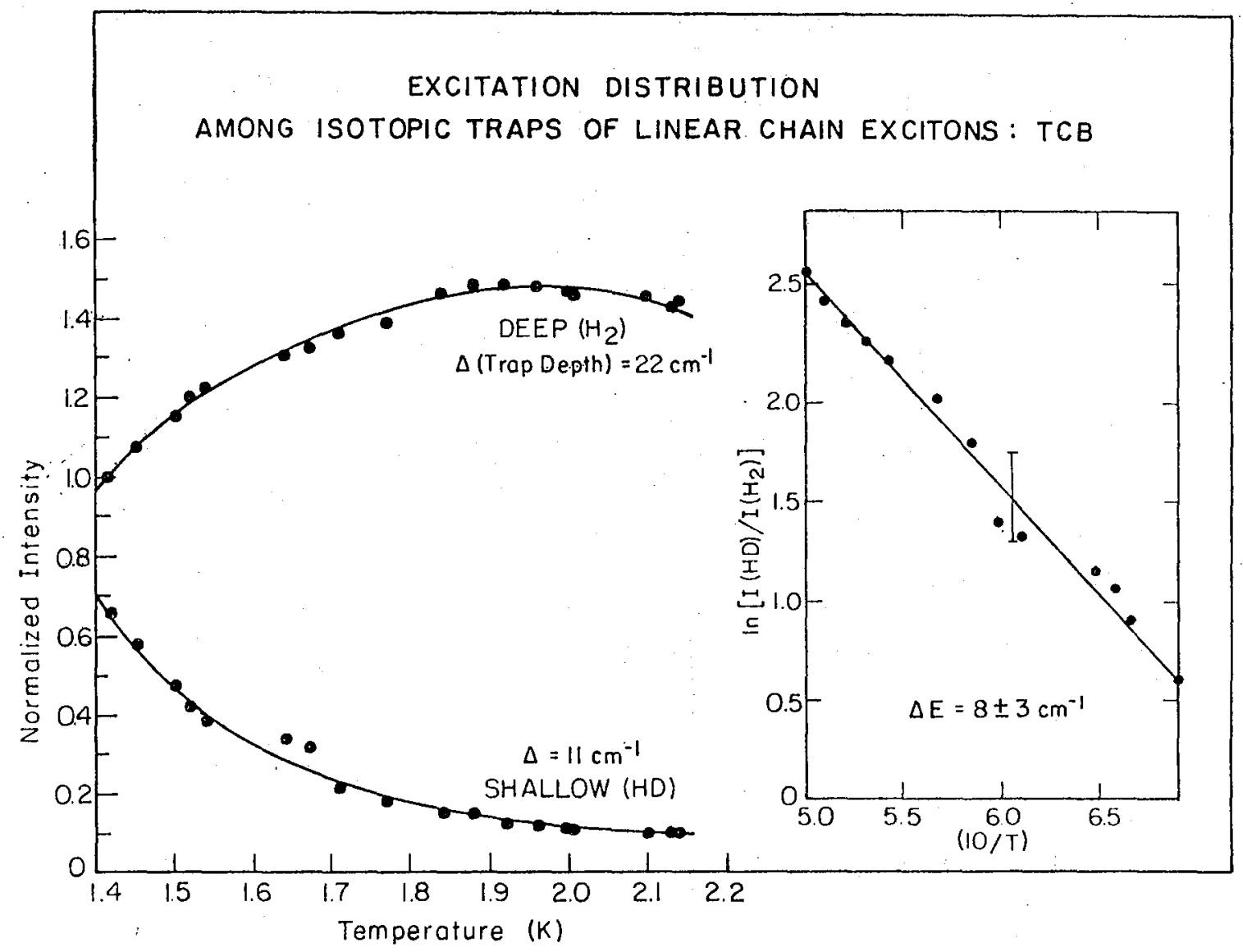

XBL. $748-6950$

Pig. 10. (A) The phosphorescence intensities of $I_{2}$ and lll) at different temperatures in the 0.06 " crystal: the behavior for temperatures above $2.15^{\circ} \mathrm{K}$ is discussed in the text. (B) The linear behavior for $\ln \left[\mathrm{I}(\mathrm{HD}) / \mathrm{I}\left(\mathrm{H}_{2}\right)\right]$ vs. $1 / \mathrm{T}$. 
i) promotion of trap population by lattice phonons to the band states of the host crystal

ii) migration via a host crystal exciton followed by iii) retrapping in a different trap state.

The validity of this model was strengthened by another series of experiments involving the $0.06 \%$ crystal. It can be recalled from chapter One that evidence of shallow trap spin polarization being trapped in the ODMR spectrum of the deep trap of TCB-mixed crystals was experimentally observable. Furthermore, the intensity of this trapped polarization changes with temperature as shown in Fig. 11. If the natural $\log$ of the ratio of the trapped shallow trap polarization intensity to decp trap electron-spin-only transition intensity is plotted versus the reciprocal of the absolute temperature, a straight line plot results. The slope of this Iine suggests an activation energy of $\sim 10 \mathrm{~cm}^{-1}$ for the process of shallow trap spin polarization appearing on the deep trap spectrum. These observations were made in the temperature range between $1.50^{\circ}$ and $2.17^{\circ} \mathrm{K}$, and as briefly mentioned in Chapter One, there is some ambiguity in the spectra obtained at lower temperatures. Taken in conjunction with the temperature dependence of phosphorescence spectra, however, these ODMR data provide further justification for the indirect model of energy transfer at low temperatures.

For the $5^{\circ}$ crystal only the emission of the deep $\left(h_{2}\right)$ trap was observed from the phosphorescence origin over the temperature range from . $1.4^{\circ}$ to $2.1^{\circ} \mathrm{K}$. Moreover, the emission intensity remained essentially constant over this range. This would imply that excited state population is partitioned effectively, owing to the high concentration, into the 
$00+04844300$

$-33-$

TEMPERATURE DEPENDENCE OF DEEP TRAP MONITORED SHALLOW TRAP SPIN POLARIZATION

D-IEI TRANSITION

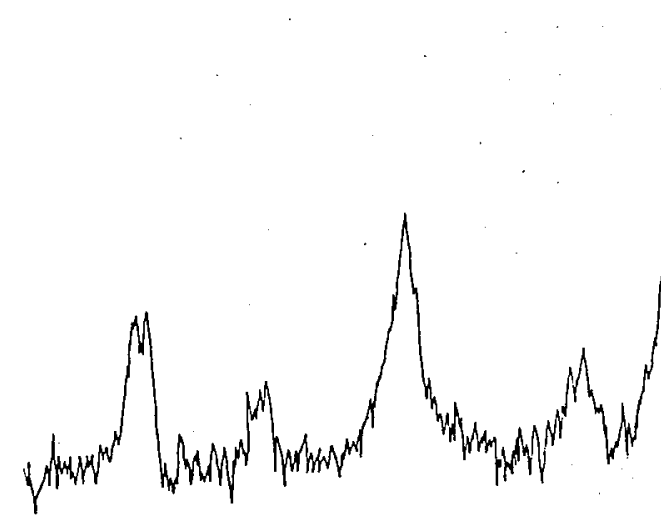
Wh $T=1.95^{\circ} \mathrm{K}$

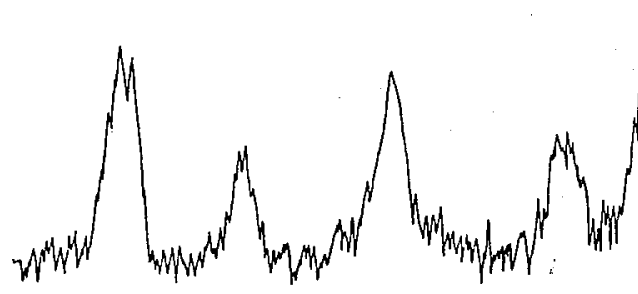

th $=1.85^{\circ} \mathrm{K}$

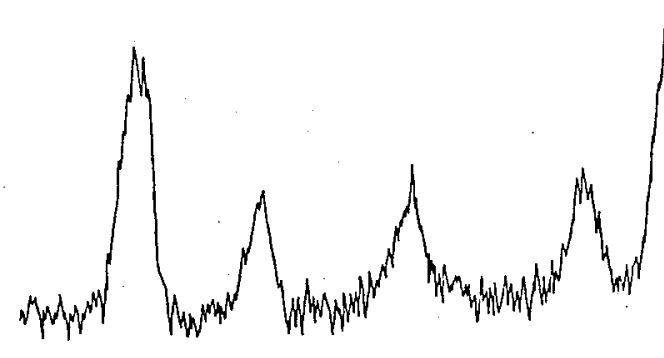

why $T=1.75^{\circ} \mathrm{K}$

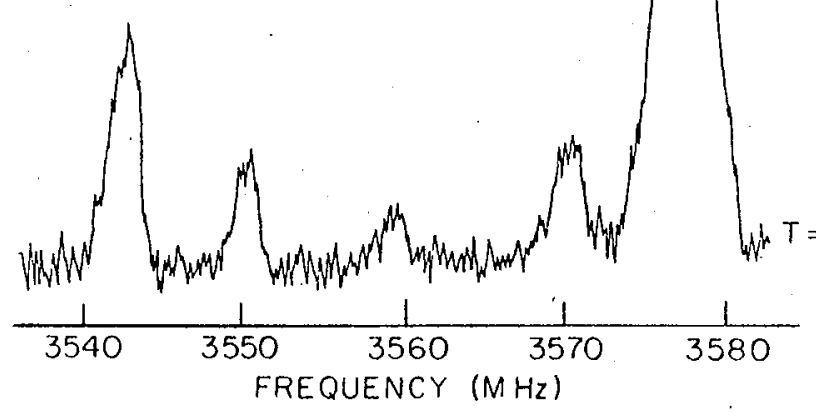

XBL776-5 599

Fig. 11. The temperature dependence of the deep trap-monitored shallow trap polarization at $3560 \mathrm{MHz}$ as a function of temperature for the D-|E| transition in a $97.5 \%$ deuterated TCB-d 2 crysta1. 
deep trap over the entire temperature range.

The results of the temperature dependence of phosphorescence experiments for the $0.06 \%$ crystal were in good agreement with the previously published results ${ }^{23}$ for temperatures from $1.4^{\circ}$ to $2.1^{\circ} \mathrm{K}$. However, the present data for the $0.06 \%$ crystal for temperatures from $2.1^{\circ}$ to $4.2^{\circ} \mathrm{K}$ is qualitatively similar to the earlier data, but quantitatively the agreement is inconsistent in two respects. First, at the lambda point of liquid helium $\left(2.17^{\circ} \mathrm{K}\right)$ a sharp decrease of approximately $30 \%$ in the emission intensity of the deep trap is observed. This decrease is independent of exciting light intensity, $h_{2}$ trap concentration, the method by which the temperature was regulated, and whether or not the crystal sample was annealed or unannealed. Second, the loss of deep trap emission intensity between the lambda point and $4.2^{\circ} \mathrm{K}$ did not closely follow an exponential curve characteristic of Boltzmann equilibria but instead appeared to fall off more linearly. The scattering of the data points in this temperature range, however, is greater than that of the data presented previously. Recent results have indicated that this discrepancy is due to the onset of bubbling in liquid helium at the lambda point. 19 Nevertheless, from the results presented here it is impossible to say with certainty whether or not trap-band communication is governed by Boltzmann statistics above the lambda point of liquid helium in these samples. For this reason analysis will be confined to data collected in the "kinctic regime" 23 between $1.4^{\circ}$ and $2.1^{\circ} \mathrm{K}$. 
Dynamics of Energy Promotion: Direct and Indirect Transfer

Processes in TCB

The results of the optically detected spin locking experiments were utilized to extract values of $\mathrm{K}^{\mathrm{P}}$, the promotion rate constant, for the deep $\left(h_{2}\right)$ trap in both the $0.06 \%$ and $5 \%$ crystals as a function of temperature between $1.4^{\circ}$ and $2.1^{\circ} \mathrm{K}$. The total decay time, $\mathrm{T}_{1 \rho}$, was determined at 10 different temperatures for the D-|E| transition of the $0.06 \%$ crystal and at 11 temperatures for the corresponding transition in the $5 \%$ crystal. A typical determination of $\mathrm{T}_{1 \rho}$ at a single temperature for the $0.06 \%$ crystal is shown in Fig. 12 . Values for $K^{L}$ were calculated from experimental measurements of the 1 ifetimes of the $\mathrm{T}_{x}$ and $\mathrm{T}_{y}$ zerofield spin sublevels of the $0.06 \%$ crystal at $1.4^{\circ} \mathrm{K} .^{23}$

$\Lambda$ value for $K^{T} 1 \rho$. was obtained from spin locking diata of the $2|\mathrm{I}|$ transition of a $1 \%$ mole/mole $\mathrm{TCB}-\mathrm{h}_{2}$ trap in a durene host. 23 In this case the localized trap state 1 ies about $1460 \mathrm{~cm}^{-1}$ below the energy of the host band, ${ }^{33}$ and $\mathrm{K}^{\mathrm{P}}$ is essentially zero. ${ }^{34}$ After subtracting the contribution of $\mathrm{K}^{\mathrm{L}}$ (again determined from individual sublevel 1 ifetimes) to the total spin-locking decay rate, a value of $\mathrm{K}^{\mathrm{T}} 1 \mathrm{\rho m}=13.8 \mathrm{~s}^{-1}$ was obtained. These values for $K^{\mathrm{L}}$ and $\mathrm{K}^{\mathrm{T}} 1 \mathrm{\rho m}$ were then utilized to extract values for $K^{\mathrm{P}}$ at each temperature from values for the total decay rate, ${ }^{\mathrm{T}}{ }^{1 \rho}$, by use of Eq. (2.1). The effect of error in $\mathrm{K}^{\mathrm{P}}$ owing to the approximate values used for $\mathrm{K}^{\mathrm{L}}$ and $\mathrm{K}^{\mathrm{T}} 1 \mathrm{\rho m}$ is not expected to be great, principally because of the large value of $\mathrm{K}^{\mathrm{T}} 1 \rho$ in comparison.

The variation of $K^{P}$ with temperature reflects the extent to which phonons couple to the excitation at a given temperature. In the dilute concentration limit the promotion of excitation from the localized 
(shallow or decp) traps into the band could involve a state which is in "quasiresonance" with the dense manifold of stationary band states, $|k\rangle$. The latter are primarily responsible for the decay to the band, since the recurrence probability is relatively small because of the high density of band states. In such cases the probability per unit time for the interaction of a localized state $|L\rangle$ into $|k\rangle$ states is

$$
P=(2 \pi / \hbar) \sum_{k}\left|V_{L K}\right|^{2} \delta\left(E-E_{k}\right)
$$

It is the nature of $\left|V_{L K}\right|^{2}$ which determines the explicit form of the perturbation for the promotion process. If the detrapping probability is not $k$-selective, the density of exciton states at energy $\Delta$ above the trap energy can be invoked into Expression (2.2). For example, if the trap-exciton interaction is via an intermediate state $|I\rangle$ and a phonon $X(\varepsilon)$ of energy $\varepsilon$, Eq. (2.2) takes the form:

$$
\begin{aligned}
P_{k}^{(\varepsilon)}= & (2 \pi / \hbar)\langle n(\varepsilon)\rangle_{T} \mid\left\langle\operatorname{LX}(\varepsilon)\left|V_{L I}\right| \operatorname{IX}(\varepsilon-\Delta)\right\rangle \\
\times & \left.\left\langle\operatorname{IXX}(\varepsilon-\Delta)\left|V_{I k}\right| k X(\varepsilon-\Delta)\right\rangle\right|^{2} \rho(\Delta) .
\end{aligned}
$$

The important thing to note is that the temperature dependence is explicitly given in the phonon distribution term (occupation number and phonon density of states), and that the problem is now somewhat simplified assuming that $\left\langle V_{I K}\right\rangle$, which is a radiationless decay matrix element, and $\left\langle V_{L I}\right\rangle$ are temperature independent. The energy of the phonon (or phonons for a multiphonon process) must cither match the chergy $\Delta$ (direct process) or exced it (Raman process); hence, the total detrapping probability will depend on the sum over all phonons and also intermediate states which match the required energy. 


\section{$00+0+604362$}

In the low temperature limit, the Planck distribution function 35 takes a simple form:

$$
n(T)=\frac{1}{e^{\varepsilon / k T}-1} \approx e^{-\varepsilon / k T} \quad
$$

Thus, in the limit where the bandwidth is small compared to $\Delta$, as in the case of TCB crystals (see Chapter One) or for selective detrapping with $\varepsilon=\Delta$, the detrapping rate constant is given by 23

$$
K^{(T)}(L+k)=A e^{-\Delta / k T} \rho(\Delta) \equiv K^{P},
$$

where $A$ is a constant. This expression is valid in the limit where the density of phonon states is almost constant, e.g. in the linear range of the dispersion for acoustic phonons. 2

Expression (2.5) shows the importance of at least two parameters in considering detrapping in crystals at low temperatures: the density of states function and the phonon distribution.

The above result was shown for the TCB system in different ways. In the kinetic regime, ${ }^{23}$ where no thermal equilibrium exists between the localized and band states, the shallow trap excitations to the band are more frequent than those of the deep traps. Consequently, at intermediate or low temperatures, the thermal steady state dynamics depend upon the tenperature variation of the band group velocity and the detrapping rate constant from the shallow trap. 23 for a one-dimensional, narrow band ICB crystal, the change in group velocity with temperature is rather small. Hence, in the limit where $K_{S}$ (total rate constant for relaxation to the ground state from the shallow trap) is smal1 compared to the detrapping rate constant, the ratio of the steady state populations of the shallow and decp traps is 23 


$$
\frac{\left(N_{S}\right)}{\left(N_{D}\right)}=\frac{\left[\left(C_{S} / C_{D}\right) K_{D}\right]}{\Lambda \rho} \times e^{+\Delta_{S} / k T}
$$

where $C_{S}$ and $C_{D}$ are the concentration of the shallow and deep traps, respectively, $K_{D}$ is the total rate constant for relaxation to the ground state from the deep trap, $\Lambda$ is the constant associated with Eq. (2.5), and $p$ is the exciton density of states. Therefore, the ratio of phosphorescence intensity of the shallow and deep traps as a function of inverse temperature should give $\Delta_{S}$, the energy separation between the shallow trap and the exciton band, as is indeed shown experimentally (see Fig. 10). Moreover, the "inverse Boltzmann" behavior is explained.

The second important feature is that Eq. (2.5) indicates that if ln $\mathrm{K}^{\mathrm{P}}$ (obtained from onsL experiments) is plotted versus $1 / \mathrm{T}$, a straight line should be obtained, the slope of which reveals the energy separation, $\Delta$, between the trap state and the final state connected by the promotion process. Plots of $1 n \mathrm{~K}^{\mathrm{P}}$ versus $1 / \mathrm{T}$ for the experimental ODSL results on the $0.06 \%$ and $5 \%$ crystals are given in Fig. 12. It can be noted that the In $\mathrm{K}^{\mathrm{P}}$ versus $1 / \mathrm{T}$ plots are essentially linear for both the $0.06 \%$ and 5\% samples (see Fig. 12 caption). More importantly, the differing slopes of these graphs indicate that promotion processes are occurring to different final states in the two crystals.

The slope of the data for the $0.06 \%$ crystal yields a value for $\Delta$ of $17 \pm 3 \mathrm{~cm}^{-1}$. This value is on the order of the optical separation between the deep trap and the host band. Thus, for the $0.06 \%$ crystal, where the density of host $\left(\mathrm{d}_{2}\right)$ band states is high, both the phosphorescence and ODSL results indicate that trap-to-trap communication is occurring predominately by an indirect process through the host band states. It 


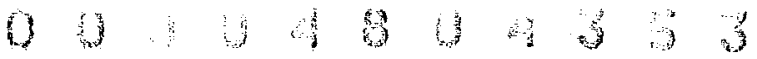

$39-$

Fig. 12. (A) A schematic for the spin-locking sequence and the hypothetical phosphorescence response: $\tau_{S L}$ is the length of time the spin-locking field is on: $S$ denotes saturation, and $i$ denotes inversion of population between spin sublevels. $\Delta \mathrm{I}$ denotes the change in phosphorescence intensity associated with the population of the coherent state after the time $T_{S l}$. (B) A typical decay of a spin-loched spin ensemble of the $0.06 \%$ crystal at $1.84^{\circ} \mathrm{K}$. (C) and (D) show plots of $K^{\mathrm{P}^{\prime}}$ versus $1 / \mathrm{T}$ for the $0.06 \%$ and $5 \%$ crystals, respectively. The values for $\triangle E$ were obtained from a computer-generated least squares fit of the experimental data to the best exponential, $\mathrm{K}^{\mathrm{P}}=\operatorname{Aexp}\left(-\Delta \mathrm{E} / \mathrm{K}^{\prime} \mathrm{I}\right)$, with each $\mathrm{K}^{\mathrm{P}}$ data point weighted in inverse proportion to the magnitude of its error. (This error is reflected in the error bar associated with each data point in the figure.) The parameters $A$ and $\Delta E / k$ obtained from the least squares fit were used to generate theoretical values for $\mathrm{K}^{\mathrm{p}}$ corresponding to each value of the temperature at which experimental measurements were made. In linear plots of $1 n k^{P}$ versus $1 / k T$, the standard deviation of the slope associated with the experimental data points from that associated with the theoretical points was determined to be $2 \mathrm{~cm}^{-1}$ using standard statistical analyses. It is felt, however, that an error of $\pm 3 \mathrm{~cm}^{-1}$ more accurately reflects the experimental error. 
PHOSPHORESCENCE RESPONSE TO SPIN LOCKING FIELD: PROMOTION CONSTANTS FOR TRAPS OF ISOTROPICALLY MIXED. TCB CRYSTALS

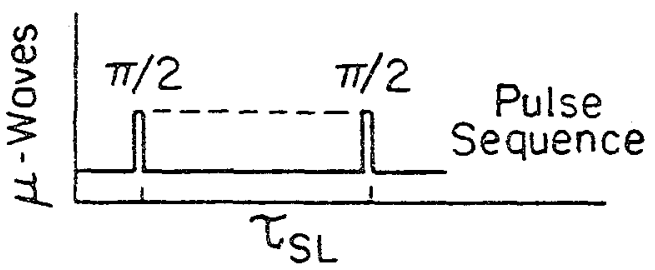

(A)
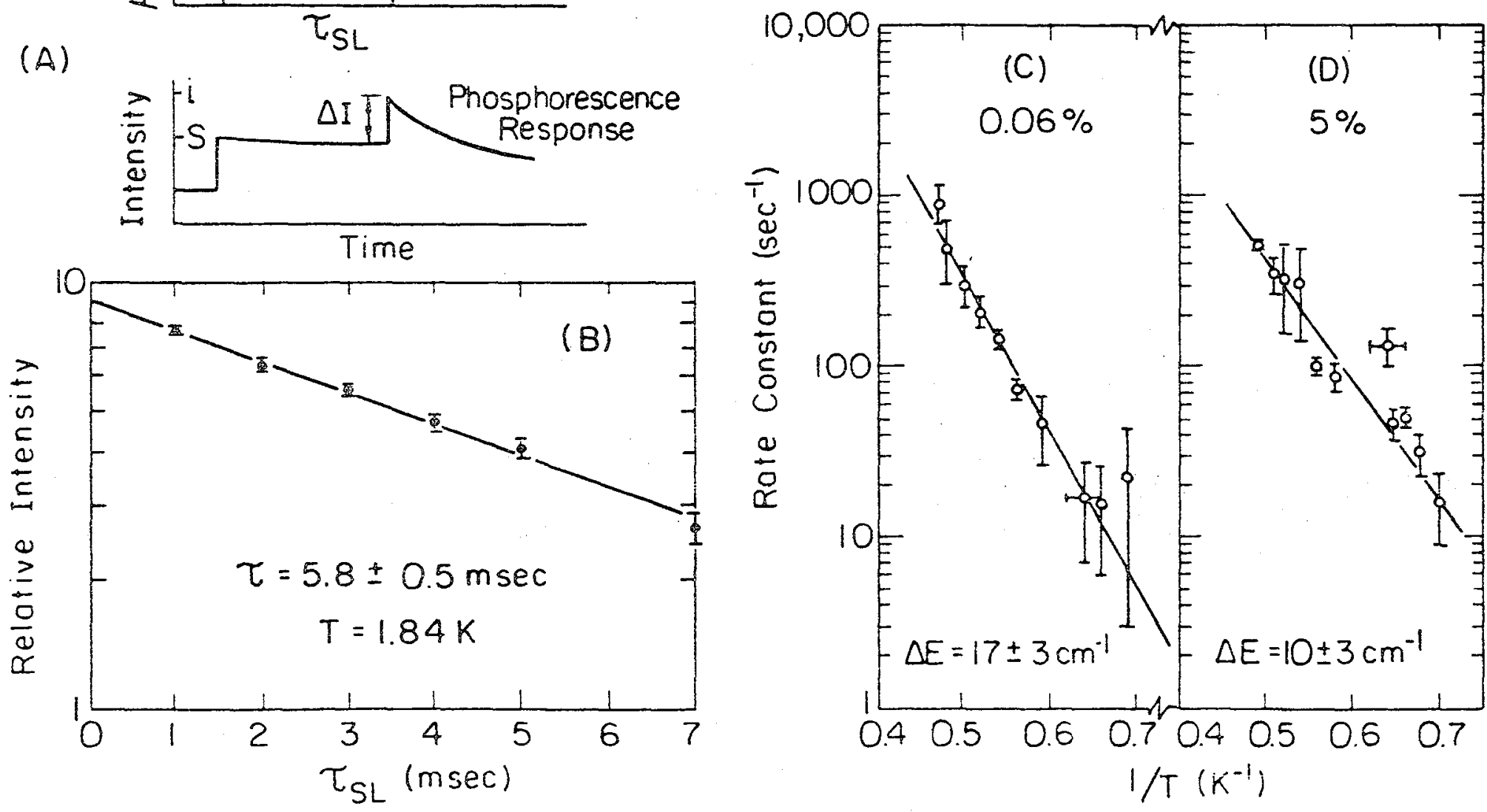

$X B L 755-1405$ 


\section{$004+404384$}

$.41-$

might be added that subsequent experiments on $99.7 \%$ deuterated TCB-d 2 crystals, where the shallow trap concentration is only $\sim 1 \%$, have yielded similar activation energies for promotion out of the deep trap. ${ }^{14}$

The slope of the data for the $5 \%$ crystal, however, yields a value for $\Delta$ of $10 \pm 3 \mathrm{~cm}^{-1}$ which is approximately the same as the scparation between the deep and shallow traps. This result points toward the predominance of direct processes in the $5 \%$ crystal. Nonetheless, it must be noted from the phosphorescence results that any population promoted out of the deep and into the shallow trap is returned by a rapid process, either by a direct or indirect route, to the deep trap. This is essentially why the $5 \%$ crystal shows only $\mathrm{TCB}-\mathrm{h}_{2}$ emission and no TCB-hd emission at steady state.

Excitation Yiclds and Transfer Times in Isotopically Mixed Crystals

There has been much interest in mechanisms for long range transfer and in transfer times in molecular systems. ${ }^{9,22,36}$ In clusters of isotopic impurities located below a one-dimensional band the transfer time can be determined through perturbation theory from a knowledge of the trap depth, the resonance interaction, and the distance between traps. 9,37 Such a treatment of trap-to-trap "tunneling" has also met with success for isotopically mixed crystals of benzene where the crystal structure is not one-dimensional. $^{36}$ Conventional optical ${ }^{9,22}$ and magnetic resonance experiments $^{38}$ have elucidated much about the detailed mechanisms important in energy transfer problems, but quantitative data about the kinetics and pathways of these transfer processes have been difficult to determine. The beaty of the optically detected spin-locking experiment is that it 
can give these transfer times directly and analytically. In addition, if the triplet spins are adiabatically demagnetized in the rotating frame 39,40 in zerofield ${ }^{13}$ and the kinetics of the demagnetized state are compared to the kinetics of the spin-locked state, additional information such as the effective quantum yield for mobile band excitons can be determined. ${ }^{41}$ This quantum yield has been found to vary from 1 to 0.1 depending on the temperature which in turn determines the degree of scattering of the mobile excitons by the phonons. 41

For a one-dimensional crystal, one possible model for treating the direct transfer process involves the phonon-assisted tunneling mechanism. 36 Here the transfer time, $t$, can be approximately related to the number of intervening host lattice sites, $n$, using perturbation theory:

$$
n=\left[\log \left(t / t^{\prime}\right)\right][\log (\Delta / \alpha \beta)]^{-1}
$$

where $t^{\prime}=\hbar / \alpha \beta$, the effective nearest neighbor transfer time, and $\alpha$ is a measure of the fraction of localized states which are successful in transferring energy, and $\beta$ is the resonance interaction energy. The difference in energy between the traps is provided by the phonon reservoir. Knowing $t\left(1 / K^{\mathrm{P}}\right)$ from spin-locking kinetics, a value for $\mathrm{n}$ can be determined. From Chapter One, $\beta=0.3 \mathrm{~cm}^{-1}$ for the $\mathrm{TCB}-\mathrm{d}_{2}$ system. If it is assumed that $\alpha \cong 1$, then this approach predicts $n$ to be on the order of 5 to 7 . It should be noted that the composition of the $5^{\circ}$ crystal gives a statistical separation between traps of approximately 10 molecules on avorage, in reasonable agreement with the theoretical prediction. The above results were derived by considering only the in-chain interactions since the interaction between chains is very small, being approximately $1 \mathrm{MHz}{ }^{42}$ 


\section{$00+3+80+393$}

$.43-$ :

Additiona1 Considerations

Application of the ODSL technique to study the kinetics of promotion from the shallow trap has not been mentioned since experiments of this sort have been precluded by an inability to coherently drive population between two spin sublevels of the shallow trap. This would indicate that transfer out of the shallow trap is occurring at a rate of at least $2-3$ $\mathrm{MHz}$ which is roughly the $\mathrm{yll}_{1}$ value for the microwave fields cmployed in the pulsed coherence experiments. Here $\gamma$ is the electron magneto-gyric ratio and $\mathrm{H}_{1}$ is the field strength. Another manifestation of the $2-3 \mathrm{MHz}$ transfer rate is the lack of quadrupole satellite structure on the shallow trap ODMR spectrum. The electron spin-nuclear quadrupole transitions occur only because the electron-nuclear hyperfine interactions mix the zerofield spin sublevels. ${ }^{12}$ Because this hyperfine coupling constant is on the order of $1 \mathrm{MHz}$, transfer processes occurring at comparable or faster rates tend to average $37,43,44$ the hyperfine interaction to zero, thus serving to eliminate the quadrupole satellites from the trap ODMR spectrum.

This much more effective promotion, in contrast to that out of the deep trap, can be explained by the presence of a greater number of phonons or combinations of phonons of appropriate energy $\left(\Delta=11 \mathrm{~cm}^{-1}\right)$ in the phonon reservoir. It is also likely that the temperature independent terms in Expression (2.5) for $K^{P}$ are different for the shallow trap since the shallow trap wavefunctions would be expected to exhibit more "band-1ike" characteristics than those for the deep trap.

The kineties of the trapping process are less well understood for the isotopically-mixed crystal system. From laser excitation experiments ${ }^{20}$ 
on the exciton band of neat TCB-h $\mathrm{h}_{2}$ crystals between $1.25^{\circ}$ and $2.00^{\circ} \mathrm{K}$, Shelby, Zewail, and Harris measured build-up times on the order of $1-10$ ms for phosphorescence from the TCB $x$-traps located $17 \mathrm{~cm}^{-1}$ below the band. Similar experiments on $\mathrm{TCB}-\mathrm{d}_{2}$ mixed crystals have not yet been performed since the presence of two different trap states complicates the interpretation of the data. It is clear, at least from the temperature dependence of phosphorescence experiments, that the two isotopic trap states are not in thermal, i.e. Boltzmann, equilibrium in the temperature range from $1.4^{\circ}$ to $2.1^{\circ} \mathrm{K}$

An alternative approach to this problem is to consider the trapband system as a two level system with the band "level" weighted by an appropriate density of states function. The problem of energy exchange between the two levels can then be treated in terms of the exchange theory developed by Anderson ${ }^{44}$ and Kubo and Tomita. ${ }^{45}$ Each 1evel has associated with it a Larmor frequency, $w$, with the difference in Larmor frequencies between the levels equal to $\delta \omega$. From the results in chapter One, $\delta \omega \sim 17 \mathrm{MHz}$. If the exchange between the two sites or levels is slow, that is if $\delta \omega \tau>1$ where $\tau$ is the lifetime in the upper or exciton level, then the ODMR transitions associated with each site should be distinct. If, however, the exchange is fast, $\delta \omega \tau \ll 1$, and only a single transition at some weighted average frequency should be observable. Therefore, as the temperature is raised and the energy exchange begins to quicken, a frequency shift should be observed in the trap ODMR spectrum. Such observations were recently reported for a two site exchange involving the lowest triplet state of p-benzoquinone and a higher-lying vibronic level. 46 
$-45-$

For the case of the $0.06 \%$ mixed crystals of TCB, no measurable shifts were observed in either the shallow or deep trap resonance frequencies over the temperature range 1.4 to $2.1 \mathrm{~K}$. This fact indicates that $\delta \omega \tau \geqslant 1$ and sets a lower 1 imit on $\tau$ of $\sim 6 \times 10^{-8} \mathrm{~s}$. Nonctheless, it is clear that this problem deserves further investigation. 


\section{CHAPTER THREE}

\section{USE OF OPTICALLY DETECTED MAGNETIC RESONANCE}

\section{AS A PROBE OF THE DIMENSIONALITY OF}

ENERGY TRANSFER IN PYRIMIDINE SINGLE CRYSTALS

Introduction

Establishing the dimensionality of molecular crystals is very important in determining the nature of energy migration. The preceding chapters have served to underline the importance of the one-dimensional nature of 1,2,4,5-tetrachlorobenzene in facilitating a straightforward link between theory and experiment. This link is, of course, critical if a thorough understanding of energy transport in molecular solids is to be.

obtained. A first approach to the problem of establishing crystal dimensionality often involves an examination of the $x$-ray crystal structure for evidence of the lamellar stacking mentioned in the Introduction. In molecular crystals where there are two or more molecules in the unit cell it is also necessary to consider the importance of the relative magnitudes of translationally equivalent and translationally inequivalent interactions, as in Fig. 13.

Using Davydov theory ${ }^{2}$ and assuming on $1 y$ nearest neighbor interactions to be important, the following dispersion relation can be derived for an anthracene-like crystal structure (monoclinic $\mathrm{P} 2{ }_{1} / \mathrm{a}$ ):

$$
E \pm(k)=2 \beta_{a} \cos (k a)+2 \beta_{b} \cos (k b)+2 \beta_{c} \cos (k c) \pm 4 \beta_{a b} \cos \left({ }^{1} k a\right) \cos \left({ }_{2}^{1} k b\right)
$$

Here the $\beta_{i}$ give the magnitude of the interaction along each of the three axes $(i=a, b, c)$, and $\beta_{a b}$ is the magnitude of the translationally inequivalent interaction between molecules along the $a$ and $b$ axes. The Davydov 


\section{RESONANT ENERGY EXCHANGE ROUTES BETWEEN COHERENTLY COUPLED MOLECULES}
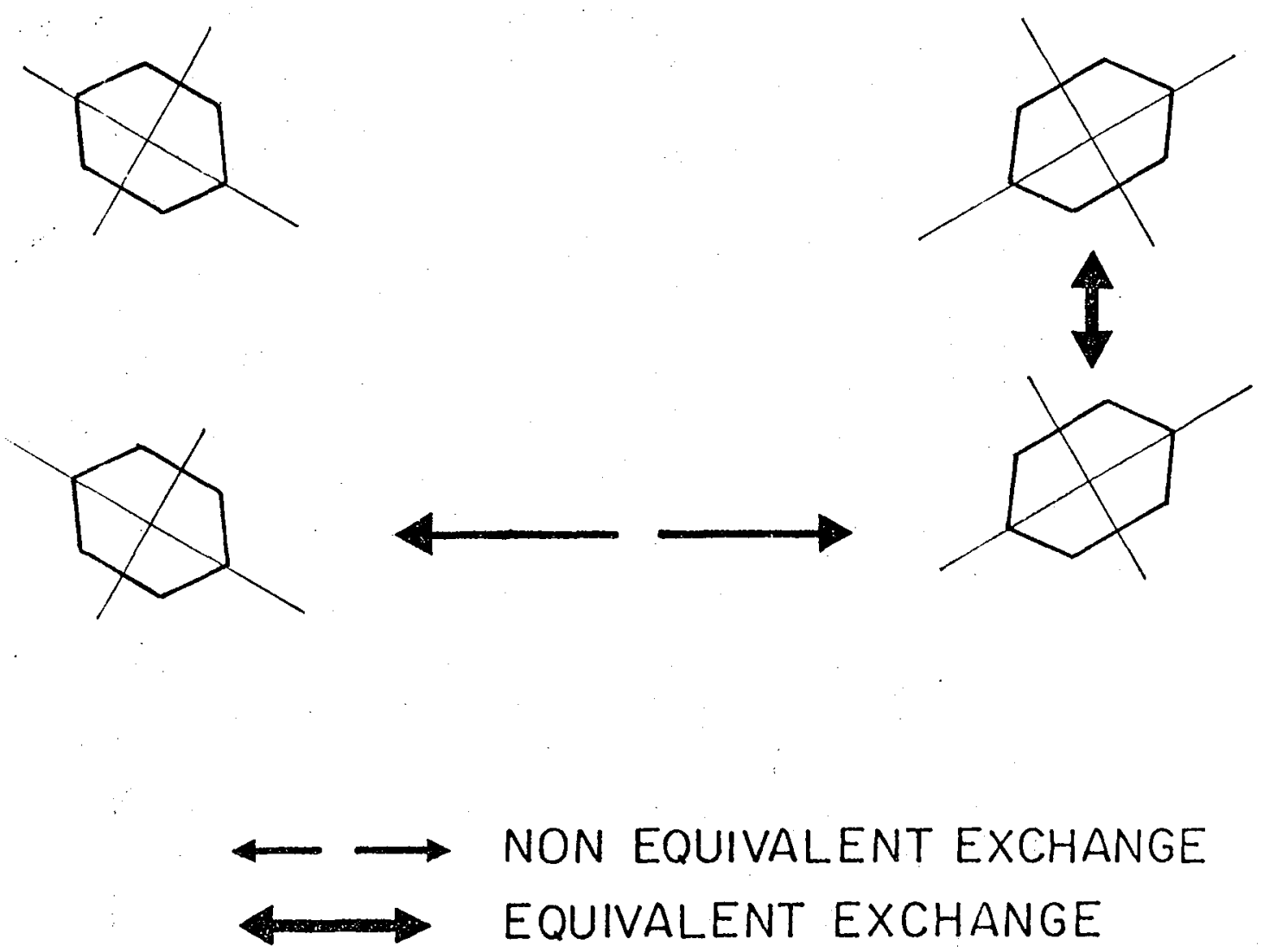

$X B L 761-6329$

rig. 13. The relationship between translationally equivalent and translationally inequivalent (non-equivalent) energy exchange in a molecular crystal. 
splitting ${ }^{2}$ is equal to $8 \beta_{a b}$. Now, the criteria for one-dimensionality are satisfied when the absolute magnitude of one of the $\beta_{i}{ }^{\prime} s$ is much greater than those for the other two, and $\beta_{a b} \cong 0$. This is the case for TCB. 9,10 If the Davydov splitting becomes measurable while one translationally equivalent interaction lominates the other two, then the molecular crystal may be described as nearly one-dimensional, as is the case for phenazine. ${ }^{47}$ Multidimensionality results when the translationally inequivalent interaction dominates the other interactions in the crystal or when two or more of the largest interactions are equivalent. 48

Pyrimidine has a crystal structure ${ }^{5,48}$ which strongly suggests that energy transfer in pyrimidine crystals should be one-dimensional. Furthermore, the singlet-triplet absorption spectrum for the lowest triplet state of pyrimidine shows no measurable Davydov splitting. ${ }^{49}$ The dimensionality of energy transport in such a case will then depend on the relative magnitudes of the translationally equivalent interactions along each of the three axes. The crystal structure suggests, however, that the interaction along the $\mathrm{c}$ axis should dominate.

In order to measure the sign and magnitude of $\beta_{c}$ in pyrimidine, Hochstrasser and Whiteman ${ }^{48}$ performed an absorption experiment utilizing crystals containing about $70 \%$ pyrimidine $-\mathrm{h}_{4}$ in a pyrimidine $-\mathrm{d}_{4}$ host. This gives a crystal with a pyrimidine-h "sublattice" consisting predominately of shorter chains (dimers, trimers, tetrimers, etc.). Because of the electric dipole $\Delta k=0$ selection rule, the $k=0$ states of each type of $\mathrm{N}$-mer could be reached in a $\mathrm{T}_{1} \leftarrow \mathrm{S}_{0}$ absorption experiment. Therefore, by observing the energy separation between each of these lines and the neat crystal $0-0$ absorption 1 ine, $\beta_{c}$ could be mcisured (assuming 


\section{$00+404438$}

unidimensionality). For pyrimidine these experiments ${ }^{48}$ resulted in a smearing out of the exciton band exclusively to higher energy of the infinite chain giving $\beta<0$ and $4 \beta \sim 15-30 \mathrm{~cm}^{-1}$. These experiments were hampered, to a large degree, by the weak oscillator strength of the $T_{1}+S_{0}$ absorption. From these results it is possible to say that the bandwidth is produced by one more translationally equivalent interactions of the same (negative) sign. This does not prove unidimensionality, but as mentioned previously, unidimensionality is strongly predicted by the crystal structure. It is obvious that another experimental technique is necessary to resolve this problem.

One possibility is the application of the ODMR techniques mentioned in Chapter One. The results of experiments using these techniques could then be compared to those expected on the basis of the simple, one-dimensional, nearest-neighbor interaction model. Direct band-to-band ODMR experiments ${ }^{10}$ are precluded by the relatively weak phosphorescence from the lowest triplet state. Observation of coherent exciton trapping ${ }^{11}$ would be dependent on obtaining isotopically-mixed pyrimidine-d ${ }_{4}$ crystals with a relatively low trap concentration. An interesting alternative to these approaches is the ODMR spectra of dimer trap states in isotopicallymixed crystals. $17,18,37,50$ This technique has been successfully applied to the one-dimensional crystals of 1,4 -dibromonaphthalene 37 and $1,2,4,5-$ tetrachlorobenzene $17,18,50$ as well as to the nearly one-dimensional crystal system of phenazine. ${ }^{47}$

Dimer spectroscopy has two principal advantages over other ODMR techniques: i) The phosphorescence intensity problem is reduced since emission is monitored from trap states which account for nearly all of 
the phosphorescence intensity at low temperatures. ii) The theory for energy transfer is simplified since a dimer is just a two-molecule exciton chain. The resonance interaction between the two translationally equivalent molecules forming the dimer splits the triplet state of the dimer into two levels separated from each other by an amount $2 B$ (compared with $4 \beta$ for an infinite chain exciton band) and from the monomer by $\beta$. Anisotropy in the spin-orbit coupling to the txiplet spin sublevels would also cause a difference in the zerofield splittings between the two dimer states, again analogous to the exciton case ${ }^{50}$ as shown in Fig. 14. Therefore, if this anisotropy is great enough, it should be possible to observe distinct ODMR spectra for each of the dimer states and the monomer. Of course, the trap concentration must be high enough $(\sim 5-10 \%)$ to assure a reasonable number of dimers, and phosphorescence must occur from all three states. For the case of translationally equivalent dimers in one-dimensional systems, only one of the dimer wavefunctions will have a non-zero transition moment to the ground state. 50 The energy difference between the zerofield splittings of the dimer states (labelled + or - ) and the monomer is related to the intermolecular interaction term $\beta$ by the same reduction factor mentioned in Chapter One,

$$
\mathrm{I}(+t)-\mathrm{E}= \pm B \mathrm{f}
$$

It is important to note at this point that an anisotropy in the lalmor frequencies of the two dimer (or, more generally, exciton) states can also be induced by a mixing between the plus and minus (or k) states via a magnetic Hamiltonian. 50 This effect is not important for translationally equivalent dimers of centrosymetric molecules since the magnetic axes of each monomer comprising the pair are parallel to each other as well as to 
01,4304359

$-51-$

THE RELATIONSHIP BETWEEN EXCITON AND N-MER DISPERSIONS IN ONE-DIMENSIONAL CRYSTAL
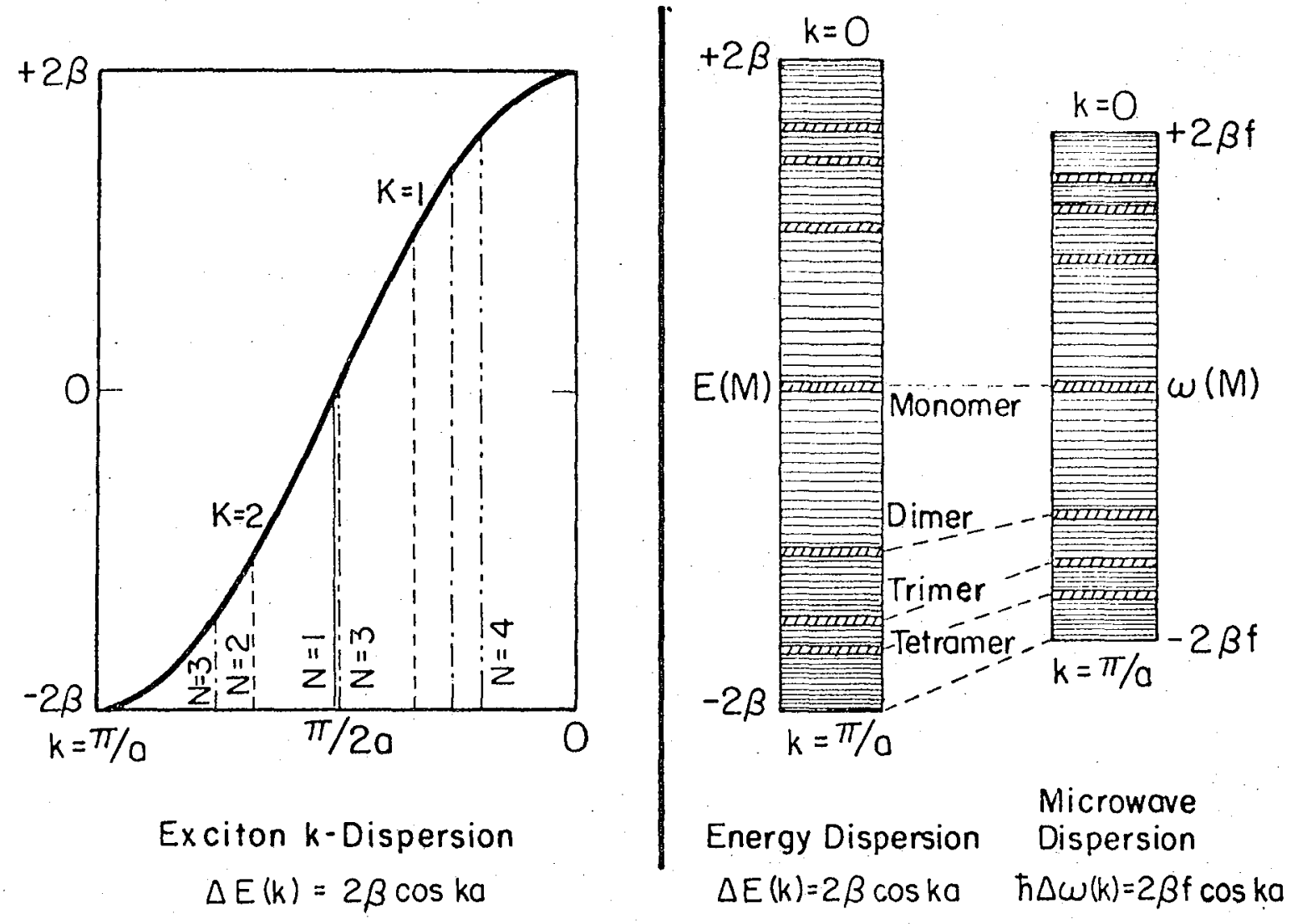

$$
\begin{gathered}
\text { N-mer } k \text {-Dispersion } \\
\Delta \epsilon(K)=2 \beta \cos (k \pi / N+1) \\
k=1, \cdots N
\end{gathered}
$$

XBL 747-6804

lig. 14. The relationship between exciton and $N$-mer dispersions ${ }^{50}$ (N.B. dimer) for a one-dimensional system. The figure on the left gives the $k$ dispersion for the exciton while the vertical lines give the energy position of the $\mathrm{N}$-mers. The rest of the $K$ states are not shown for the sake of clarity. The schematic on the right-hand side of the figure gives both the energy and microwave dispersions for the different states of the different clusters. The figure clearly shows that there is a one-to-one correspondence between the optical and microwave dispersions. 
those of the dimer. In crystals where there is no inversion symmetry between the molecules comprising the pair, however, this mechanism could become important. ${ }^{37,51}$ This can best be seen by considering the dimer wavefunctions and Hamiltonian.

The wavefunctions for a triplet state dimer can be constructed from the appropriate spin-orbit functions for each molecule in the pair and are given by 50

$$
\psi_{i}( \pm)=(1 / \sqrt{2})\left(\left|\Phi_{A}^{*} T_{i} \Phi_{B}^{o} S^{o}\right\rangle \pm\left|\Phi_{A}^{o} S^{o} \Phi_{B}^{*} T_{i}\right\rangle\right)
$$

Here $\Phi_{A}$ and $\Phi_{B}$ represent the orbital part of the wavefunctions for the two molecules, $A$ and $B$, which comprise the dimer. The asterisks denote the molecule being excited to its lowest triplet state. The $\mathrm{T}_{\mathrm{i}}$ represent the zerofield triplet spin functions, and $S^{\circ}$ refers to the singlet ground state spin function. These six basis functions can then be used to obtain the dimer energies for any value of the intermolecular potential $V_{A B}$. A general matrix element for the dimer Hamiltonian in this basis can then be written 50

$$
\left\langle\psi_{i}( \pm)\left|H_{A}+H_{B}+V_{A B}+H_{S}^{A}+H_{S}^{B}\right| \psi_{j}( \pm)\right\rangle=\delta_{ \pm \mp}\left(-\delta_{i j} D_{i j} \pm\left\langle V_{A B}\right\rangle\left\langle T_{i}^{A} \mid T_{j}^{B}\right\rangle\right)
$$

$\mathrm{H}_{\mathrm{A}}+\mathrm{H}_{\mathrm{S}}^{\mathrm{A}}$ is the monomer Hamiltonian (space + spin) for molecule $\mathrm{A}$ and similarly for molecule $B$. The $D_{i j}$ are the fine-structure tensor elcments. It can be noted that for translationally equivalent dimers composed of centrosymmetric molecules, the zerofield splittings of the diner and monomer should be identical in the absence of an anisotropy in the spinorbit coupling since $\left\langle\mathrm{T}_{i}^{\mathrm{A}} \mid \mathrm{T}_{j}^{\mathrm{B}}\right\rangle=\delta_{i j}$. For molecules like pyrimidine 


\section{0}

(symmetry $\mathrm{C}_{2 v}$ ), however, $\left\langle\mathrm{T}_{i}^{\mathrm{A}} \mid \mathrm{T}_{j}^{\mathrm{B}}\right\rangle \neq \delta_{i j},{ }^{51}$ leading to a possible difference in monomer and dimer Larmor frequencies. In addition, when the triplet principal axes of the monomer and dimer are not paralle1, transfer of energy back and forth between the dimer molecules would provide a modulation of the fine-structure axes and would lead to spin-lattice relaxation.

Of course, the magnitude and degree of such effects will depend on the values of the spin projections $\left\langle\mathrm{T}_{i}^{\mathrm{A}} \mid \mathrm{T}_{j}^{\mathrm{B}}\right\rangle$. For example, for the case of 1,4-dibromonaphthalene, ${ }^{37}$ which has a one-dimensional crystal structure but lacks a molecular center of symmetry, there was no measurable difference found in the zerofield splittings of the monomer and dimer. Nevertheless, these factors need to be taken into account if the ODMR spectra of dimers are to be used in studying the dimensionality of energy transfer in pyrimidine.

The remainder of this chapter will be concerned with the application of coherent dimer spectroscopy to studies of isotopically-mixed pyrimidine crystals.

\section{Experimental}

Preparation of pyrimidine- $\mathrm{d}_{4}$ was carried out using a modification of the method of smith and christensen. 52 2,4,5,6-Tetmalhoropyrimidine (Eastman) was dissolved in anhydrous ether and was exchanged with 99.d deuterium gas over a $10^{\circ} \mathrm{Pd}$ on charcoal catalyst using a Parr hydrogenerator with the initial $\mathrm{D}_{2}$ gas pressure set at roughly $3 \mathrm{~atm}$. The reaction flask also contained a sufficient amount of $20 \% \mathrm{NaOH}$ in $\mathrm{D}_{2} \mathrm{O}$ to neutralize the DC1 evolved during the course of the reaction. Twenty percent NaOD 
in $\mathrm{D}_{2} \mathrm{O}$ was also utilized, but 1 ittle difference was noted in the isotopic content of the final product. Following uptake of the theoretical amount of $D_{2}$, the reaction flask was again pressurized to $3 \mathrm{~atm}$, and the exchange was continued for an additional 3-4 hours. The reaction mixture was then filtered, and the residue was washed with several portions of hot $\mathrm{n}_{2} 0$. The filtrate was then cooled to $-5^{\circ} \mathrm{C}$, and the aqueous portion of the filtrate was saturated with NaOll. This mixture was kept at $-5^{\circ} \mathrm{C}$ for about 12 hours. Following this, the ether portion was removed, and the aqueous portion was extracted with five portions of anhydrous ether. The ether-pyrimidine solutions were combined and dried over anhydrous $\mathrm{Na}_{2} \mathrm{SO}_{4}$ for 24 hours. After drying, the ether-pyrimidine solution was filtered through activated charcoal, and the ether was removed by rotoevaporation. The pyrimidine product was further purified using two different methods. The first method involved repeated (four) vacuum distillations. In the second approach the pyrimidine was vacuum-distilled into a zone-refining tube which was sealed under $\sim 200$ microns pressure of dried $\mathrm{N}_{2}$ gas. This pyrimidine was then zone-refined for 150 passes at 10 passes/day in a $-15^{\circ} \mathrm{C}$ cold room, and the middle portion of the zonerefined material was removed for use in experiments. Both methods gave pyrimidine (melting point $21^{\circ} \mathrm{C}$ ) of high purity as confirmed by massspectral analysis.

The pyrimidine-d 4 prepared by this method, however, showed on $1 y \sim 91^{\circ}$ deuteration. This low percentage of deuteration in spite of the high isotopic purity of the deuterium gas used in the synthesis probably resulted from the use of a hydrogenation apparatus which had been used extensively for hydrogen exchange and which contained a substantial amount 
of adsorbed $\mathrm{H}_{2}$ which could not be efficiently removed by the methods available. The mass spectrum showed a crystal composition of approximately $68 \%$ pyrimidine- $\mathrm{d}_{4}, 25 \%$ pyrimidine $-\mathrm{d}_{3}$ h (presumably with the proton substituted in the 2 position on the ring), $6 \%$ pyrimidine-d ${ }_{2} h_{2}$, and $1 \%$ pyrimidine-dh ${ }_{3}$. No detectable (>0.1\%) pyrimidine-h ${ }_{4}$ was observed. Pyrimidine-h 4 (Aldrich) which was used as a dopant was purified by repeated (three) vacuum distillations.

Crystals were prepared by mixing appropriate amounts of the $91 \% \mathrm{~d}$ pyrimidine- $\mathrm{d}_{4}$ with the $\mathrm{h}_{4}$ dopant and vacuum-distilling the mixture into a crystal growing tube. Single crystals were grown from the melt using a Bridgman furnace which was placed in a $-15^{\circ} \mathrm{C}$ cold room. The crystals were cut, polished, and mounted in a slow-wave helix; the process being carried out in the $-15^{\circ} \mathrm{C}$ cold room. The mounted crystals were then transferred in an ice-water-salt bath to the liquid helium dewar which had previously been cooled with liquid nitrogen. This process preserved the crystal quality to a large degree.

The benzene utilized as a host matrix was purified by zone refining $M C B$ Spectroquality benzene for 100 passes at 10 passes/day in a $-15^{\circ} \mathrm{C}$ cold room. The benzene from the middle portion of the zone-refined material was then used in the preparation of pyrimidine-doped single crystals. Crystal preparation was identical to that used for the isotopica11y-mixed pyrimidine crystals.

Continuous wave and pulsed ODNR experiments were carried out as discussed previously. Excitation was accomplished by light from an Osram HBO 100-watt $\mathrm{Ig}$ arc lamp filtered at $2800 \AA$. Excitation at this wavelength populated the second excited singlet state of pyrimidine from 
which the lowest triplet state is fairly efficiently populated by spinorbiting coupling. 53 Phosphorescence decay times were measured by appropriately shuttering the exciting light and recording the decays on a Northern NS-575 signal averager. Laser excitation experiments on the lowest triplet state were performed using a Chromatix CMX-4 flash lamp-pumped dye laser and frequency doubling on the emission from an oxazine 170 perchlorate (Eastman) dye solution which had an average power at 30 pps of $\sim 60 \mathrm{~mW}$ at $7000 \AA$.

\section{RESULTS AND DISCUSSION}

\section{The Lower Excited States of the Pyrimidine Molecule}

The lower electronic levels of pyrimidine are shown in Fig. 15. 54 The characterization of these levels has, for the most part, been carried out on "isolated" pyrimidine molecules in benzene or cyclohexane host crystals. These two hosts have proved to be particularly good since experimental evidence suggests that the guest pyrimidine molecules assume predominately one substitutional site in the hosts. It can be scen that the general rule ${ }^{55}$ that spin-orbiting coupling is stronger between different orbital types $\left(n \pi^{*}\right.$ and $\pi \pi^{*}$ ) than between like types is followed by pyrimidine. Ilence, only the $\mathrm{T}_{y}$ and $\mathrm{T}_{z}$ triplet sublevel states can be significantly involved in the spin-orbit coupling process.

Burland and Schmidt ${ }^{54}$ have done a very careful study of the triplet state propertics of pyrimidine- $\mathrm{h}_{4}$ and pyrimidine-d 4 in benzene and cyclohexane hosts utilizing ODMR techniques. Their results are summarized in Table 1. It can be noted that the lifetime measurements confirm the group theoretical predictions noted in Fig. 15. N1so, the similarity between 
THE LOWER ELECTRONIC LEVELS OF PYRIMIDINE

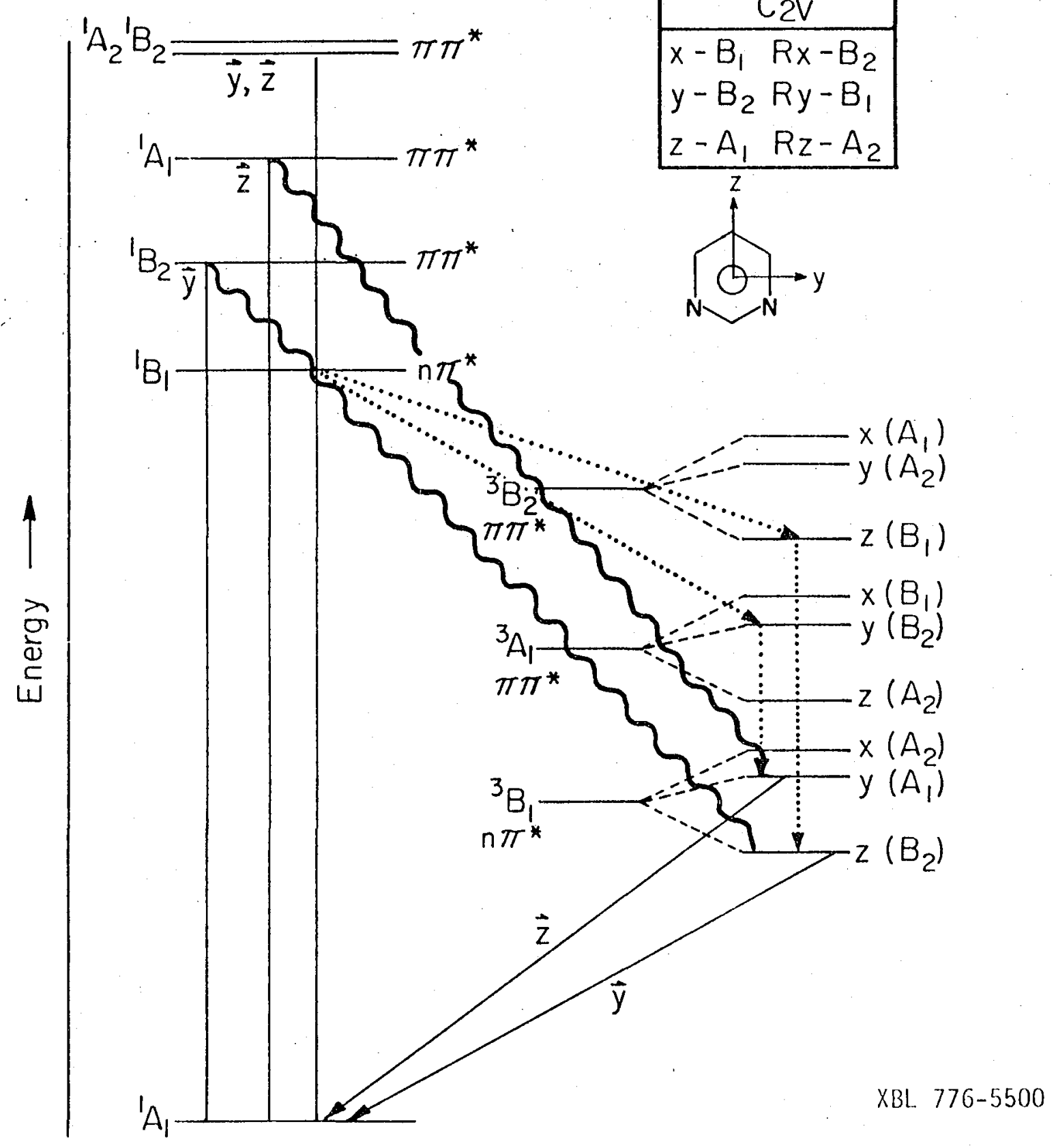

Fig. 15. The lower electronic energy levels of pyrimidine. ${ }^{54}$ The vertical scale only represents the general ordering of the encrsy levels. The symmetry labels in parentheses refor to the total symmetry (space $\otimes$ spin) of the triplet sublevels. The ordering of these sublevels is only known for the lowest triplet state, ${ }^{3} B_{1}$. Solid lines connecting encrgy levels indicate radiative transitions with their corresponding polarizations; wavy lines connect some of the spin-orbit coupled states; and dotted lines indicate radiationless internal conversion and intersystem crossing processes. 
TABLE 1. Pyrimidine zerofield splitting parameters.

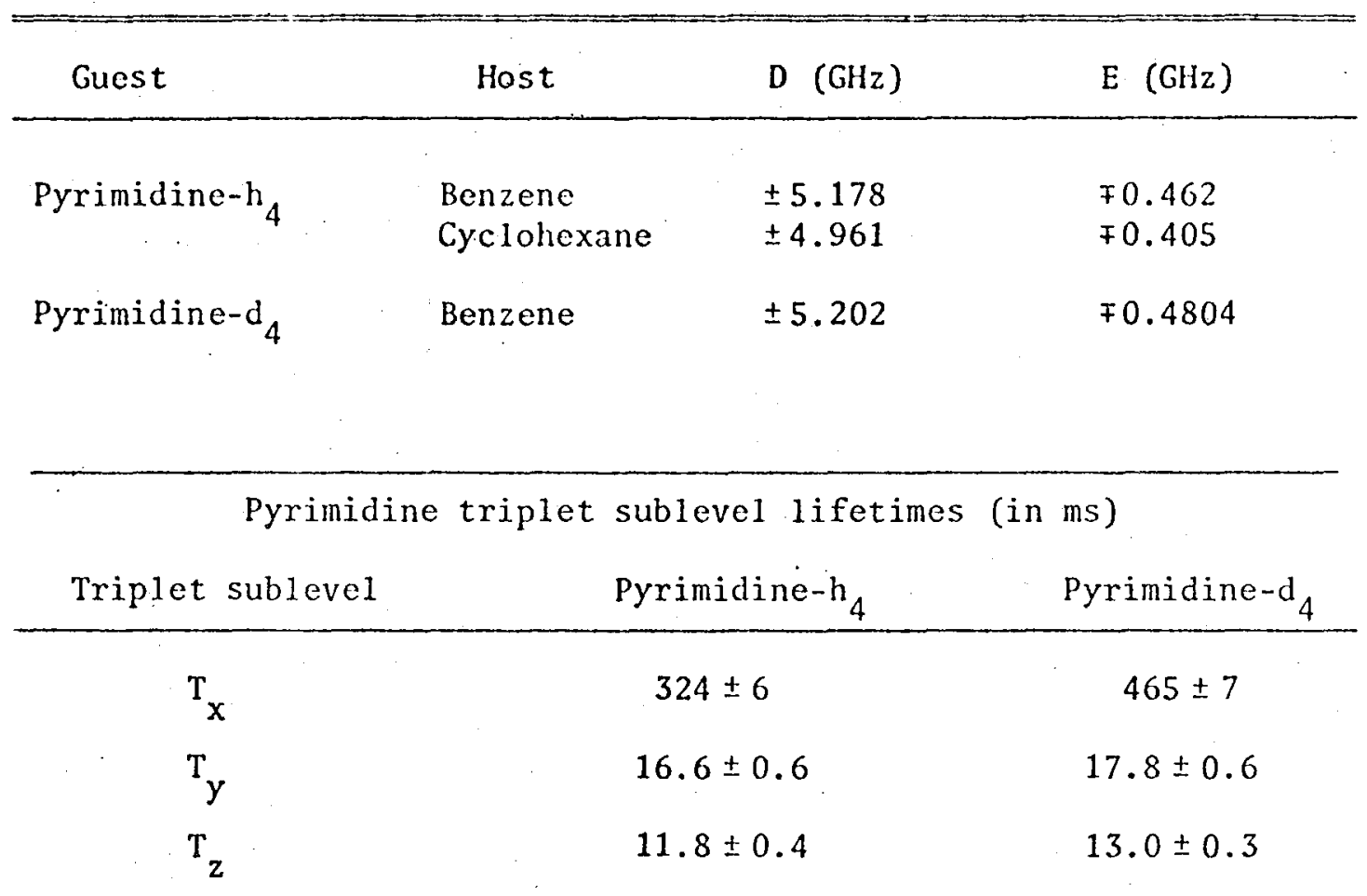




\section{$00 \times 4404363$}

$-59$

the 1 ifetimes of the $h_{4}$ and $d_{4}$ isomers indicates that the levels decay primarily via radiative processes, with the radiative strength divided about equally between the $T_{y}$ and $T_{z}$ sublevels. These results could not be completely reconciled with the Zeeman effect studies of Hochstrasser and Lin on pure pyrimidine single crystals. ${ }^{56}$ The results of these authors showed that the $\mathrm{T}_{\mathrm{z}}$ sublevel carried $93 \%$ of the radiative strength, the other $7 \%$ coming from the $\mathrm{T}_{y}$ sublevel. This discrepancy could possibly be due to differing crystal field effects in the two hosts or to the presence of pure crystal exciton states in the neat crystal. Populating rate studies for each of the three triplet sublevels of pyrimidine were also carried out by Burland and Schmidt. These studies indicated that the $T_{x}$ sublevel was substantially populated in both benzene and cyclohexane hosts contrary to group theoretical predictions. The relatively high $\mathrm{T}_{\mathrm{x}}$ sublevel population could possibly be explained by assuming that one of the higher triplet states is non-planar.

Characterization of the Isotopically-Mixed Pyrimidine Crystal System

As a first step in characterizing the lowest triplet state of the pyrimicline mixed crystal system, an attempt was made to record the $\mathrm{T}_{1} \leftarrow \mathrm{S}_{0}$ absorption spcctrum using the technique of laser excitation. This technique has proved useful in cases where the oscillator strength of the transition is very small, as has been shown for pyrimidine. ${ }^{48}$ In this approach absorption of radiation by the triplet state is monitored . by recording the resulting phosphorescence. For the excitation of the lowest triplet state of the pyrimidine mixed crystal, the output of a dye laser was scanned in the region of the pyrimidine-d 4 absorption origin 
at $3442.2 \AA\left(29043 \mathrm{~cm}^{-1}\right) .57$ Lmission (to the singlet ground state, as well as to the first excited vibrational level of the ground state) was monitored from the $d_{4}$ origin and from the origin of each of the isotopic trap species $\left(\mathrm{d}_{3} h, \mathrm{~d}_{2} \mathrm{~h}_{2}\right.$, and $\left.\mathrm{dh}_{3}\right)$. No detectable emission was observed. This lack of measurable absorption could be due either to the fact that the laser pulses lacked a sufficient number of photons for efficient absorption or to the fact that the pyrimidine mixed crystal used in these experiments afforded only a $1-\mathrm{cm}$ path length for absorption.

As a second approach to characterizing the isotopically-mixed pyrimidine system, a $0.1 \% \mathrm{w} / \mathrm{w}$ pyrimidine-d $4,-\mathrm{d}_{3} \mathrm{~h},-\mathrm{d}_{2} \mathrm{~h}_{2},-\mathrm{dh}_{3}$ in benzene crystal was prepared. Following excitation into the pyrimidine singlet manifold, a phosphorescence spectrum characteristic of pyrimidine-d 4 was observed which was identical to previously published spectra. ${ }^{57}$ In addition, the spectrum (Fig. 16) exhibited emission lines which could be attributed to the pyrimidine- $d_{3} h$ and $d_{2} \dot{h}_{2}$ species, in rough proportion to their isotopic abundance. ODMR spectra of the $\mathrm{d}_{4}$ trap site gave zerofield splittings in good agreement with those obtained by Burland and Schmidt. 54 The D+|E| transition of pyrimidine-d 4 is shown in Fig. 17. The width at half height of $\sim 4 \mathrm{MHz}$ is probably due to hyperfine interactions, and its narrowness indicates that the pyrimidine molecules occupy one substitutional site in the benzene host. The $D+|E|$ transition of pyrimidine-d ${ }_{3}$ was also observed with a similar halfwidth but with a resonimo frequency maximum $5 \mathrm{MHz}$ less than that for pyrimidine-d 4 . 
PHOSPHORESCENCE SPECTRUM OF $91 \%$ O PYRIMIDINE- $d_{4}$ IN BENZENE

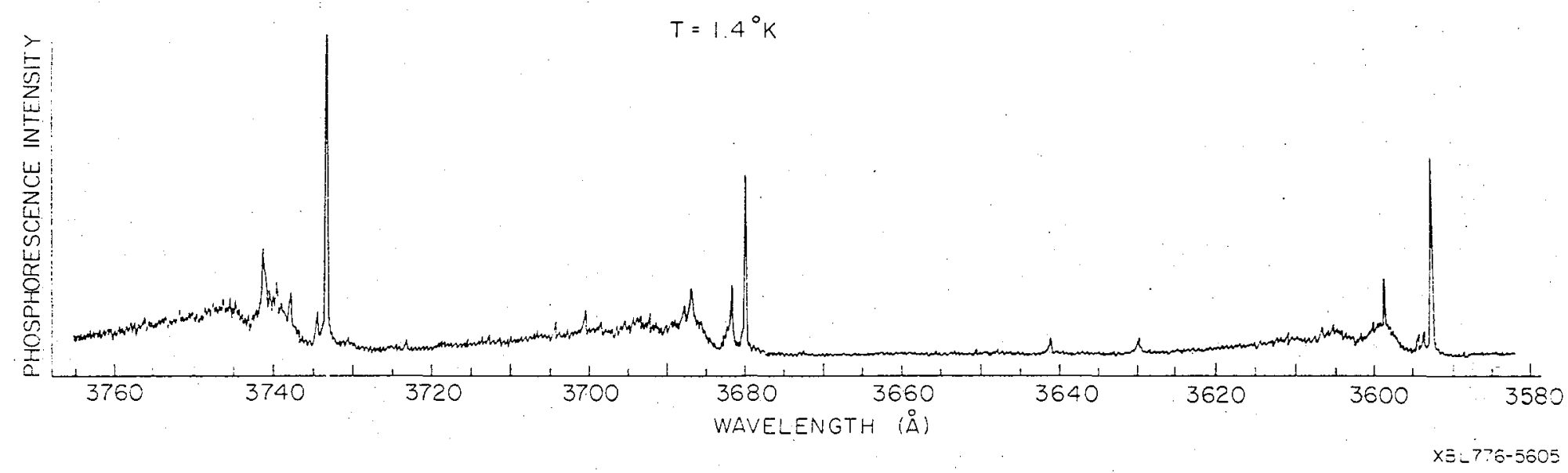

Fig. 16. Voderate resolution phosphorescence spectrum (partial) of $91^{\circ}$ d pyrimidine-d $0.1 \% \mathrm{w} / \mathrm{w}$ in benzune at $1.4^{\circ} \mathrm{K}$. 
ODMR SPECTRUM OF PYRIMIDINE- ${ }_{4}$ IN BENZENE

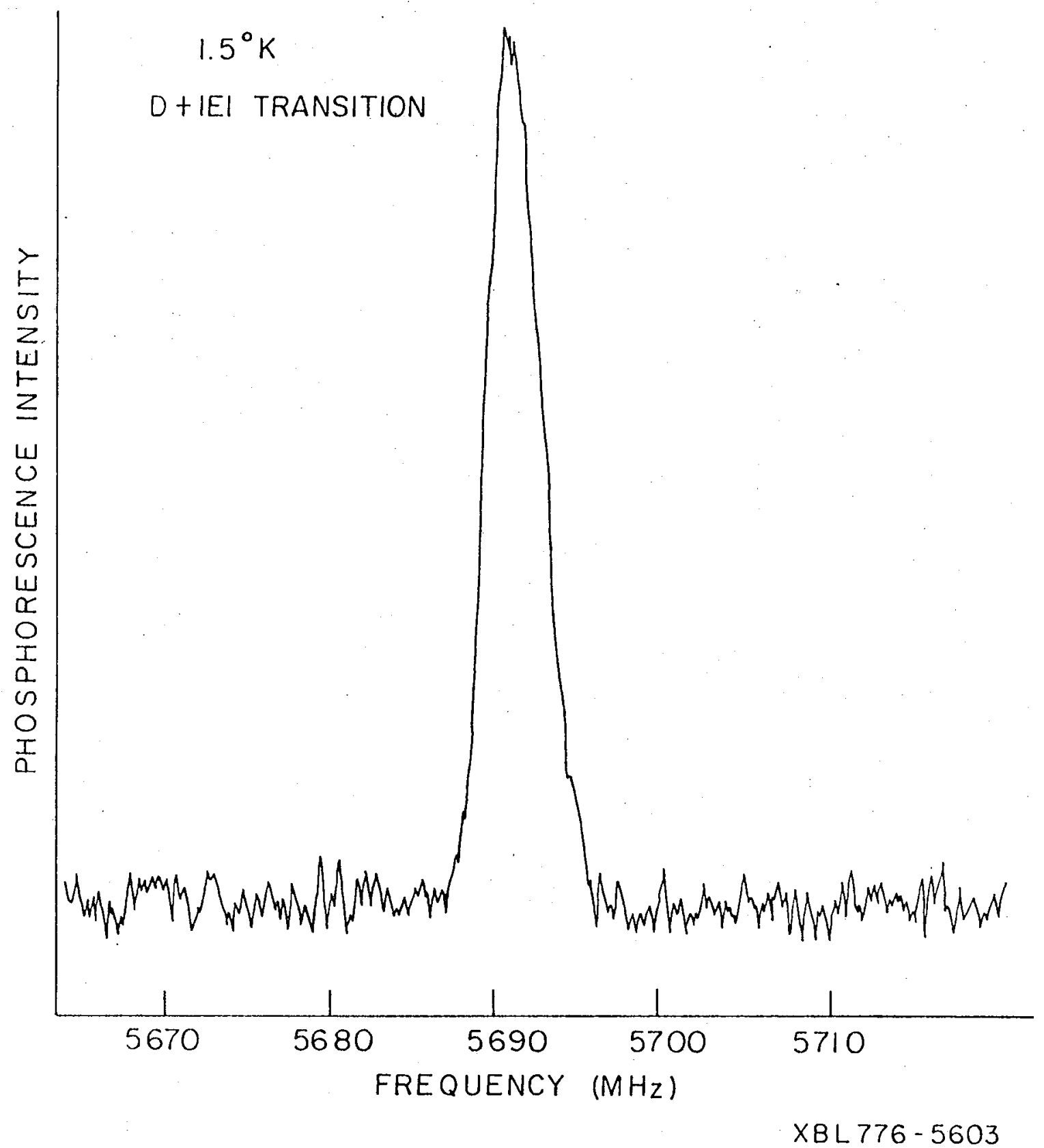

Fig. 17. Medium power $(10 \mathrm{~mW}) \mathrm{D}+\left.|\mathrm{E}|\right|_{\text {OlMR }}$ transition of pyrimidinc- $\mathrm{d}_{4}$ $0.1 \% \mathrm{w} / \mathrm{w}$ in benzene at $1.5^{\circ} \mathrm{K}$. 


\section{3}

$-6.3-$

Optical and Optically Detected Magnetic Resonance Spectra

of Isotopically-Mixed Pyrimidine Single Crystals

The phosphorescence emission spectrum of the $91^{\circ} \mathrm{d}$ pyrimidine-d 4 isotopically-mixed crystal at $1.4^{\circ} \mathrm{K}$ is shown (partial) in Fig. 18. The emission spectrum at $4.2^{\circ} \mathrm{K}$ was substantially the same. The emission origin is $\sim 3447.5 \AA\left(29006 \mathrm{~cm}^{-1}\right)$, and the spectrum for the most part consists of a few broad (10 to $15 \mathrm{~cm}^{-1}$ width at half height) transitions superimposed on a rather intense, unresolved background. The two main peaks in the emission origin are centered at roughly $3448 \AA\left(29000 \mathrm{~cm}^{-1}\right)$ and $3453 \AA\left(28960 \mathrm{~cm}^{-1}\right)$, with the first peak possibly consisting of two overlapping lines, The first vibration at $3528 \AA\left(29000-660 \mathrm{~cm}^{-1}\right)$, however, shows three well-defined lines. From the single crystal absorption studies of Hochstrasser and Marzzacco, 57 it is known that the pyrimidine-d $\mathrm{d}_{4}$ exciton absorption origin is $3442.2 \AA\left(29043 \mathrm{~cm}^{-1}\right)$ and that that for pyrimidine-h $\mathrm{h}_{4}$ is $3458 \AA\left(28908 \mathrm{~cm}^{-1}\right)$. As can be seen, the difference in the origins of the two crystals amounts to $135 \mathrm{~cm}^{-1}$. Making the rather simple assumption that the origins of the intervening isotopic species, pyrimidine $-\mathrm{d}_{3} \mathrm{~h},-\mathrm{d}_{2} \mathrm{~h}_{2}$, and $-\mathrm{dh}_{3}$, should be spaced equally gives a separation of $135 / 4 \cong 34 \mathrm{~cm}^{-1}$ between traps. This would then predict trap origin emission lines at $3447.2 \AA\left(\mathrm{d}_{3} \mathrm{~h}\right), 3451.2 \AA\left(\mathrm{d}_{2} \mathrm{~h}_{2}\right)$, and $3455.3 \AA$ $\left(\mathrm{dh}_{3}\right)$. The low temperature spectrum in $\mathrm{Fig} .18$ can then be interpreted in terms of these predictions, assuming that the $\mathrm{d}_{4}$ emission at $3442.2 R$ is too weak to be observed. The pair of overlapping lines centered at $\sim 3448 R$ could then be associated with the pyrimidine- $\mathrm{d}_{3} \mathrm{~h}$ and $\mathrm{d}_{2} \mathrm{~h}_{2}$ species while the line at $3453 . R$ could be identified as belonging to the $\mathrm{dh}_{3}$ isotopic species. If there is emission occurring from pyrimidine-h 4 , it is 


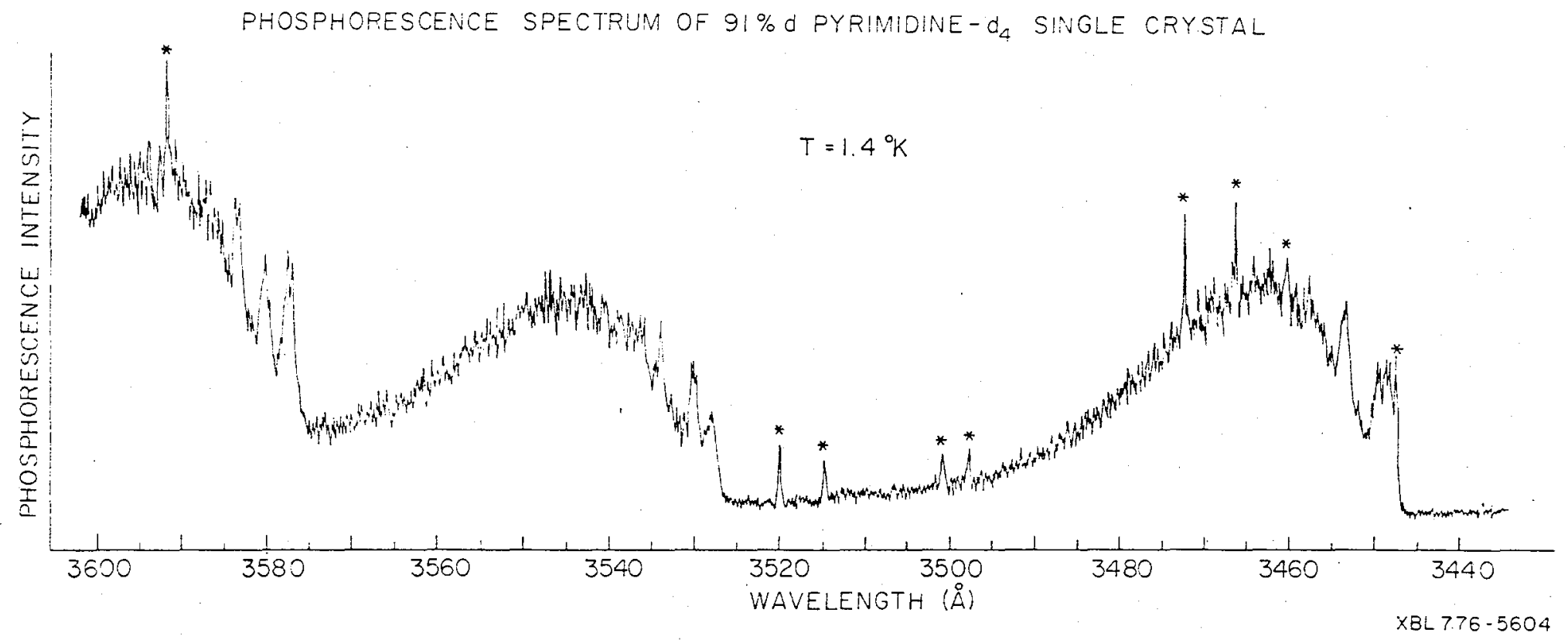

Fig. 18. Hoderate resolution phosphorescence spectrum (partial) of $91 \%$ d pyrimidine-d 4 single crystal at $1.4^{\circ} \mathrm{K}$. The lines marked with asterisks are due to a neon calibration lamp. 
probably obscured by the broad background. It must be remembered, however, that the mass spectrum of the $91^{\circ} \mathrm{od}$ crystal showed no trace $(>0.1 \%)$ of pyrimidine-h 4 . In all cases the majority of the phosphorescence from these trap states decayed with a 1 ifetime of about $22 \mathrm{~ms}$, the remainder decaying with a lifetime $>200 \mathrm{~ms}$.

Three crystals of the $91 \% d$ pyrimidine-d $d_{4}$ were prepared which contained $5.3 \%, 12.6 \%$, and $42 \%$, respectively, of pyrimidine- $\mathrm{h}_{4}$ dopant. These three crystals gave phosphorescence spectra (see Fig. 19) consisting of an emission line origin at $3458 \AA\left(28910 \mathrm{~cm}^{-1}\right)$ with a broad, unresolved emission to lower energy. The width of the pyrimidine-h 4 line was fairly broad being $\sim 10 \mathrm{~cm}^{-1}$ at half height.

The ODMR spectra for each of these crystals were obtained by monitoring the phosphorescence from each of the various trap species. Because the ODMR transitions proved to be quite weak in intensity, it was necessary to use rather wide (200 to $300 \mu)$ slit widths on the optical spectrometer. This process, which allowed for the collection of more light, reduced the resolution to a great enough extent that isolating the phosphorescence from a single molecular species was sometimes precluded. Only the D- $|E|$ transition could be observed for each of the trap species. The following values were obtained for resonance frequency maxima:

$\begin{array}{cc}\text { pyrimidine- } \mathrm{d}_{3} \mathrm{~h} & 4645 \mathrm{MHz} \\ -\mathrm{d}_{2} \mathrm{~h}_{2} & 4630 \\ -\mathrm{dh}_{3} & 4625 \\ -\mathrm{h}_{4} & 4617\end{array}$

Although the ODMR transitions were quite broad (12 to $15 \mathrm{MHz}$ widths at half height), the observable difference in resonance frequency as a function of trap depth indicates the existence of an anisotropy in the 


\section{EMISSION SPECTRUM OF \\ PYRIMIDINE $-\mathrm{h}_{4}$ TRAP ORIGIN \\ AT $1.4^{\circ} \mathrm{K}$}

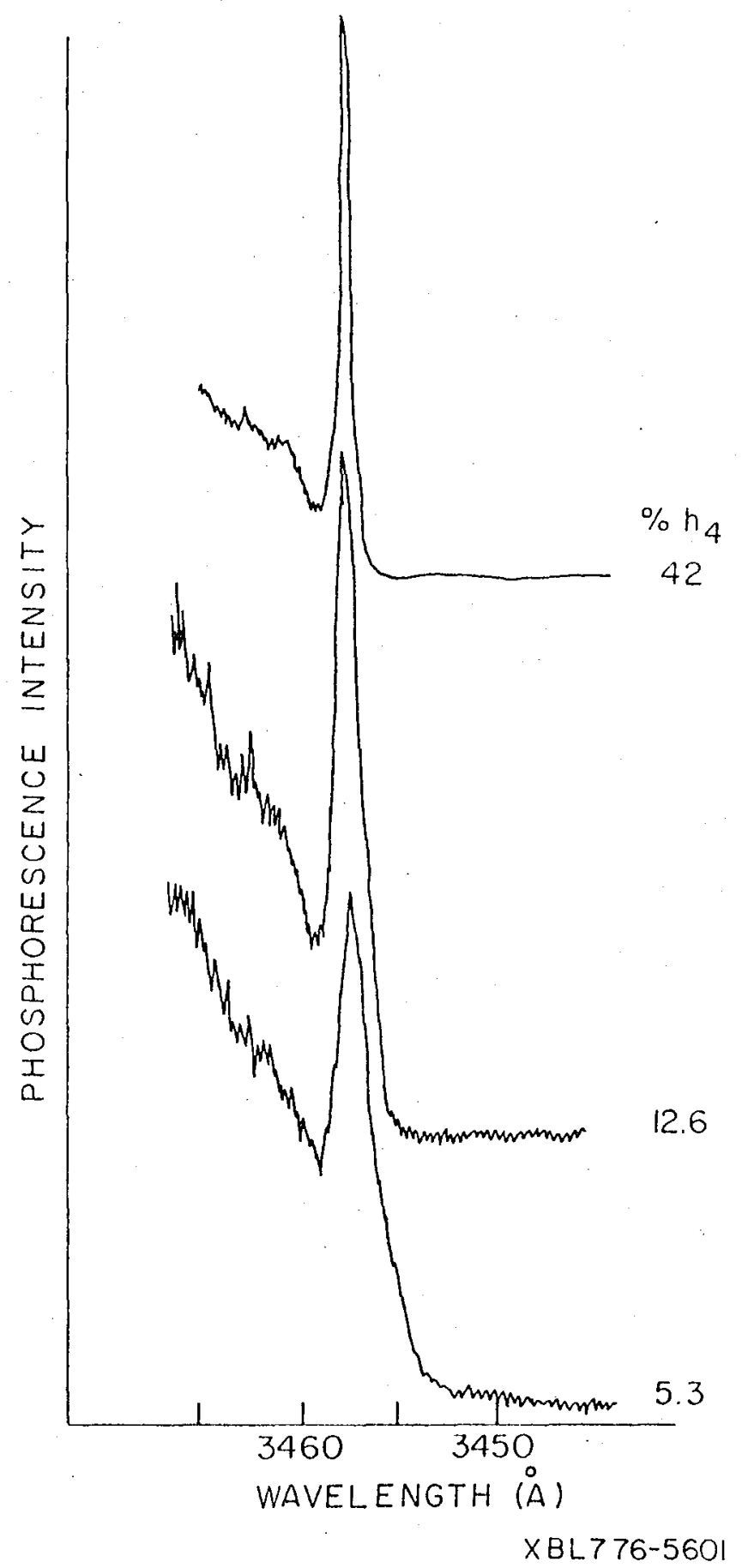

Fig. 19. Molerate resolution phosphorescence spectra of the pyrimidine-h 4 trap origin for various concentrations of pyrimidine-h $\mathrm{h}_{4}$ in $91 \% \mathrm{~d}$ pyrimidine-d 4 host crystals. All spectra were recorded under similar experimental conditions. 


\section{7}

$-67-$

spin-orbit coupling. This anisotropy would also be expected to alter the Larmor frequencies of the dimer states with respect to the frequency of the monomer.

The ODMR spectra were recorded in two ways. In the first approach the microwaves were amplitude-modulated, and the phosphorescence response was processed using a lock-in amplifier. This approach allowed the use of very high time constants but necessitated the use of very long sweep times. In the second method the spectra were recorded using rapid sweep times and very low (or no) time constants with the output of the phototube being averaged over 100 or more scans on a Northern NS-575 signal averager. These two approaches gave identical spectra.

The ODMR spectrum for the $5.3 \%$ pyrimidine- $\mathrm{h}_{4}$ in the $91 \% \mathrm{~d}$ pyrimidine-d 4 host is shown in Fig. 20. As can be seen, this spectrum has a broad, asymmetric lineshape. ODMR spectra for the pyrimidine-h 4 species were recorded at microwave power levels ranging from a few microwatts to several watts. With the exception of a slight broadening at powers greater than $\sim 1$ watt, the lineshape of the pyrimidine-h 4 D- $|E|$ transition remained unchanged, and no noticeable dimer structure could be resolved. This was true even in the $42 \% \mathrm{~h}_{4}$ crystal.

Several comments can be made concerning these results. By comparing the phosphorescence spectra of the $5.3 \%, 12.6 \%$, and $42 \%$ pyrimidine-h 4 in 91\%d pyrimidine-d crystals (lig. 19), it can be seen that the emission line of the $h_{4}$ molecule neither shifts nor broadens to any appreciable extent over the concentration range. Indeed, it seems to narrow at higher $h_{4}$ concentrations. In addition, the $h_{4}$ emission lineshape remains fairly symetrical within the confines of the $\sim 3 \mathrm{~cm}^{-1}$ resolution of the 
ZERO FIELD ODMR SPECTRUM OF ISOTOPICALLY MIXED PYRIMIDINE CRYSTALS

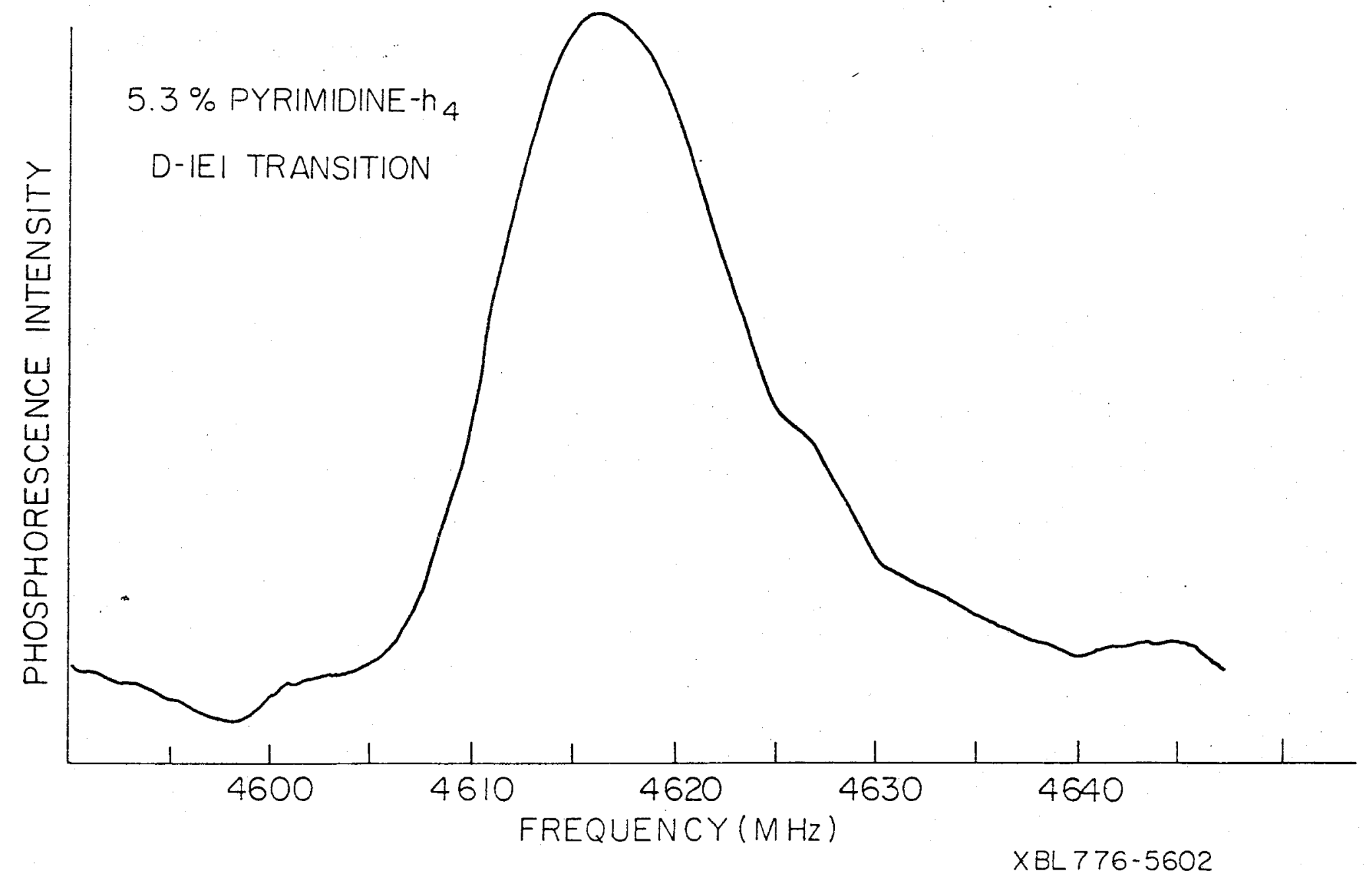

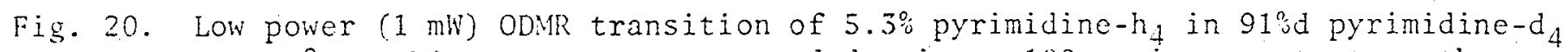
at $1.4^{\circ} \mathrm{K}$. This spectrum was recorded using a $100 \mathrm{~s}$ time constant on the lock-in amplifier and a scanning rate of $\sim 20 \mathrm{KHz} / \mathrm{s}$. 


\section{$00 \times 0430.360$}

spectrometer. Recalling the absorption experiments of Hochstrasser and Whiteman 48 mentioned earlier in this chapter, emission from pyrimidine isotopically-mixed crystals containing a reasonable number of $\mathrm{N}$-mer trap states would be expected to show an asymetric broadening of as much as $15 \mathrm{~cm}^{-1}$. The $\sim 10 \mathrm{~cm}^{-1}$ width at half height of the pyrimidine $-\mathrm{h}_{4}$ emission line would indicate, then, a value for $\beta$ on the order of $2-3 \mathrm{~cm}^{-1}$ as opposed to the somewhat larger value of $4-7.5$ predicted from the work of Hochstrasser et al. ${ }^{48}$ The rather large linewidths of the emission of the associated isotopic species in the phosphorescence spectra of the undoped $91^{\circ} \mathrm{d}$ pyrimidine- $\mathrm{d}_{4}$ crystal might also be explained in terms of the presence of phosphorescence from dimers, trimers, etc. in the emission. It is to be noted, however, that the emission line associated with the $\mathrm{dh}_{3}$ species, which by mass-spectral analysis comprises on $1 y \sim 1 \%$ of the moleules in the crystal, is also quite broad. Since pyrimidine has only $\mathrm{C}_{2 \mathrm{v}}$ symmetry, it is also necessary to consider whether or not all the different possible $\mathrm{dh}_{3}$ molccules (d in the $2,4,5$, or 6 position) will have the same zero-point energies and hence emit light of the same frequency. Similar considerations would hold for the $d_{2} h_{2}$ and $d_{3} h$ species.

The lack of resolvable dimer structure in the ODMR spectra could possibly be explained in terms of the apparent small value of $\beta\left(2-3 \mathrm{~cm}^{-1}\right)$, which would in turn lead to a smaller value for the dimer-monomer splitting. The experimental results also indicate that the effect of the lack of inversion symmetry in the dimer point group in inducing frequency differ- ences between the monomer and dimer is small. It is also possible that this lack of inversion symmetry contributes a substantial $\mathrm{T}_{1}$-type relaxation process to the dimer system, thus broadening the lines of the dimer 
ODNR spectra to a great enough degrec to make them indistinguishable from the monomer spectrum. If, indeed, pyrimidine is two-dimensional, the existence of different values of $\beta$ in the two types of chains would generate different Larmor frequencies for the dimer states in each chain. This effect could also cause the observed broadening in the ODMR spectrum. Unfortunately, it is not possible to differentiate these possible causes of broadening on the basis of the data available.

. The lack of a difference between the phosphorescence spectra of the $91^{\circ} \mathrm{d}$ crystal at $1.4^{\circ}$ and $4.2^{\circ} \mathrm{K}$ indicates that there is no efficient mechanism for thermalization of excited state population among the various trap states at these temperatures. The rather large number of isotopic "barriers" which vary in height from $\sim 34 \mathrm{~cm}^{-1}$ to $135 \mathrm{~cm}^{-1}$ no doubt serve to effectively localize excited state population on the various traps or trap chains.

The rather broad, intense emission to low energy of the trap lines appearing in the origin and in each of the vibronic transitions is probably attributable to very efficient coupling between the crystalline lattice modes (phonons) and the intramolecular excitation. Physically, lattice modes appear in the electronic spectrun for one of two reasons: ${ }^{58}$ (i) The cxcited molecule has an equilibrium disposition in the latticc which is different from the one it has in the ground state. (ii) The lattice mode perturbs the electronic transition in such a manner as to assist the clectronic motion to gain radiative properties. It is generally believed that reason (i) clominates the excited state-phonon coupling in triplet states of molecular crystals. ${ }^{59}$ In oither of the above cases it 
is essential that the coupling between the electronic motion and the lattice vibrations does not vanish. For this to be true the electron involved in the observed optical transition has to be effectively involved in the binding of the host lattice. The greater the contribution of the electron undergoing the optical transition to the binding of the crystal, the stronger the coupling between the electronic and lattice motion.

The coupling between the electronic and lattice motion in the lowest triplet state of pyrimidine is expected to be fairly strong since the excited state involves the promotion of one of the nitrogen non-bonding electrons into the ring $\pi$-system. It is primarily the $\mathrm{C}-\mathrm{H} \cdots \mathrm{N}$ hylrogen bonding which determines the molecular packing in the crystal. ${ }^{54}$ Hence, the lone-pair nitrogen electrons are involved in both the determination of the crystal lattice structure and the formation of the lowest cxcited triplet state in pyrimidine crystals. The extent of excited state-phonon coupling is underlined by comparing the enission spectra of $91^{\circ} \mathrm{d}$ pyrimidine- $d_{4}$ as a single crystal and as a guest in a benzene host matrix, Figs. 16 and 18. It can be seen that in both cases, the emission is built on a vibronic progression of roughly $656 \mathrm{~cm}^{-1}$, but it must be noted that the phonon "side-band" structure in the single crystal pyrimidine spectrum is much more intense. Similar behavior has been noted in the phosphorescence spectra of pyrazine. 58

Foglizzo and Novak ${ }^{60}$ have obtained the Raman spectrum of crystalline pyrimidine- $\mathrm{h}_{4}$ at $90^{\circ} \mathrm{K}$. Thirteen Raman-active bands were found in the region of 120 to $35 \mathrm{~cm}^{-1}$ which could be assigned to lattice modes. These lattice frequencies are in the same region as those determined for pyrazine. 58 It is very possible, then, that coupling between the electronic motion and 
phonons and/or phonon combinations is responsible for the $\sim 300 \mathrm{~cm}^{-1}$ wide sidebands observed on the pyrimidine phosphorescence spectrum. Specific assigmment of these lattice modes will have to be made before a more quantitative analysis of the spectrum can be made.

In summary, the experimental results for pyrimidine-mixed crystal systems cinnot be used to firmly establish the dimensionality of energy transfer in the first excited triplet state other than to suggest that the magnitude of the translationally equivalent interaction(s) is somewhat smaller than previously expected. Some interesting manifostations of the interplay between the electronic and lattice motions in the excited state have, however, been observed. Before more concrete information can be obtained, it will be necessary to prepare pyrimidine-d 4 crystals of a much higher deuteration percentage $\left(\sim 99^{\circ}\right)$, thus allowing the crystalline system to be better characterized. 


\section{RFIFRENCES}

1. J. Frenke1, Phys. Rev. 37, 171276 (1931).

2. A. S. Davydov, Theory of Molecular Excitons (McGraw-Hil1, New York, 1962).

3. J. Jortner, S. A. Rice, J. L. Katz, and S.-I. Choi, J. Chem. Phys. 42, $309(1965)$.

4. C. Dean, M. Pollak, B. M. Craven, and G. A. Jeffrey, Acta Cryst. 11, $710(1958)$.

5. P. J. Wheatley, Acta Cryst. 13, 80 (1960).

6. C. B. Harris and W. G. Breiland, "Coherent Spectroscopy in Electronically Excited States," in Spectroscopy: A Modern Treatise (Plenum Publishing Corp., in press).

7. M. A. El-Sayed, "Doub1e Resonance Techniques and the Relaxation Mechanisms Involving the Lowest Triplet State of Aromatic Compounds," in Excited States, Vol. 1, E. C. Lim, editor (Academic Press, New York, 1974).

8. R. M. Hochstrasser and J. D. Whiteman, J. Chem. Phys. 56, 5945 (1972).

9. R. M. llochstrasser, T. Li, H. -N. Sung, J. E. Wessel, and A. II. Zewail, Pure App1. Chem. 37, 85 (1974).

10. A. H. Francis and C. B. Harris, Chem. Phys. Lett. 9, 181 and 188 (1971).

11. A. H. Francis and C. B. Harris, J. Chem. Phys. 55, 3595 (1971).

12. M. J. Buckley and C. B. Harris, J. Chem. Phys. 56, 137 (1972).

13. W. G. Breiland, H. C. Brenner and C. B. Harris, J. Chem. Phys. 62, 3458 (1975).

14. J. C. Brock, Ph.D. Thesis, University of California, Berkeley (1976).

15. M. D. Fayer, Ph.D. Thesis, University of California, Berkeley (1974).

16. C. B. llarris and M. D. Fayer, Phys. Rev. B 10, 1784 (1974).

17. ^. H. Zewail and C. B. Harris, Chom. Phys. Lett. 28, 8 (1974).

18. A. H. Zewail and C. B. Harris, Phys. Rev. B 11, 952 (1975).

19. D. D. Dlott and M. D. Fayor, Chem. Phys. Lett. 11, 305 (1970).

20. R. M. Shelby, A. H. Zewail, and C. B. Harris, J. Chem. Phys. 64, 3192 (1976). 
21. D. [.. Dexter, J. Chem. Phys. 21, 836 (1953).

22. G. C. Nieman and G. W. Robinson, J. Chem. Phys. 37, 2150 (1962); $38,1326(1963)$.

23. M. D. Fayer and C. B. Harris, Phys. Rev. B 9, 748 (1974);

M. D. Fayer and C. B. Harris, Chem. Phys. Lett. 25, 149 (1974).

24. C. B. Harris, R. L. Schlupp, and H. Schuch, Phys. Rev. Lett. 30, 1019 (1973).

25. H. C. Brenner, J. C. Brock, and C. B. Harris, J. Chem. Phys. 60, 4448 (1974).

26. R. P. Feymman, F. L. Vernon, and R. W. Hel1warth, J. App1. Phys. $28,49(1957)$.

27. C. B. Harris, J. Chem. Phys. 54, 972 (1971).

28. A. G. Redficld, Phys. Rev. 98, 1787 (1955).

29. I. Solomon, C. R. Acad. Sci. 248, 92 (1959).

30. W. G. Breiland, C. B. Harris, and A. Pines, Phys. Rev. Lett. 30, 158 (1973).

31. The temperature was stable to approximately $\pm 0.06^{\circ} \mathrm{K}$ during the entire time of an experiment.

32. J. Schmidt, W. G. van Dorp, and J. H. van der Waals, Chem. Phys. Lett. 8, 345 (1971).

33. R. V. Nauman, Ph.D. Thesis, University of California, Berkeley (1947).

34. The rate constant $\left(K^{\mathrm{P}}\right)$ for the $\mathrm{D}-|\mathrm{E}|$ transition of TCB in a durene host has been shown to be independent of temperature (F. Ochs and C. B. Harris, unpublished results).

35. R. C. Tolman, The Principles of Statistical Mechanics (Oxford University Press, London, 1967), p.512.

36. F. B. Tulron and S. D. Colson, J. Chom. Phys. 65, 4184 (1976), and references therein.

37. R. M. Hochstrasser and $\Lambda$. 11. Zewail, Chem. Phys. Lett. 4, 142 (1974).

38. R. W. Brandon, R. E. Gerkin, and C. A. Hutchinson Jr., J. Chen. Phys. 37, 447 (1962);

N. Hirota and C. A. Hutchinson Jx., J. Chem. Phys. 42, $2869(1965)$;

N. Hirota, J. Chem. Phys. 43, 3354 (1965);

R. H. Clarke, Chem. Phys. Lett. 6, 413 (1970); 
11. Zimmerman, D. Stch1ik, and K.-H. Hausser, Chem. Phys. Lett. 11, 496 (1971);

J. Gromer, H. Sixl, and H. C. Wolf, Chem. Phys. Lett. 12, 574 (1972);

H. (.. Brenner and C. A. Hutchinson Jr., J. Chem. Phys. 58, 1328

$(1973)$;

H. C. Brenner, J. Chem. Phys. 59, 6362 (1973).

39. C. P. Slichter and W. C. Holton, Phys. Rev. 122, 1701 (1961).

40. A. (i. Anderson and S. R. Hartman, Phys. Rev. 128, 2023 (1962).

41. H. C. Brenner, J. C. Brock, M. D. Fayer, and C.. B. Harris, Chem. Phys. Lett. 33, 471 (1975).

42. See reference 33 in reference 18 .

43. H. Schuch and C. B. Harris, Z, Naturforsch. 30a, 361 (1975).

44. P. H. Anderson, J. Phys. Soc. Japan 9, 316 (1954).

45. R. Kubo and K. Tomita, J. Phys. Soc. Japan 9, 888 (1954);

R. Kubo, ibid., 935 (1954).

46. C. A. Van't Hof and J. Schmidt, Chem. Phys. Lett. 42, 73 (1976).

47. A. H. Zewail, Chem. Phys. Lett. 33, 46 (1975).

48. R. M. Hochstrasser and J. D. Whiteman, in Organic Scintillators and Liquid Scintillation Counting, D. L. Horrocks and C.-T. Peng, editors (Academic Press, New York, 1971), p.511.

49. Reference 7 in reference 48 .

50. A. H. Zewail and C. B. Harris, Phys. Rev. B 11, 935 (1975).

51. R. M. Hochstrasser and 'T. -S. Lim, J. Chem. Phys. 49, 4929 (1968).

52. V. H. Smith and B. E. Christensen, J. Org. Chem. 20, 829 (1955).

53. B. J. Cohen and L. Goodman, J. Chem. Phys. 46, 713 (1966).

54. I. N. Burland and J. Schmidt, Mol. Mhys. 22, 19 (1971).

55. K. K. Innes, J. P. Byrne, and I. G. Ross, J. Mol. Spec. 22, 125 (1967).

56. Reference 26 in reference 54 .

57. R. M. Hochstrasser and C. J. Marzzacco, J. Mo1. Spec. 42, 75 (1972).

58. M. A. M1-Sigred and W. R. Moomaw, in Lixcitons, Magnons and Mhonons. Mol. Cryst. Proc. Int. Symposium 1968, $\Lambda$. B. Zahlan, editor

(Cambridge University Press, Cambridge, 1968), p.103. 
59. R. M. Hochstrasser and P. N. Prasad, in Lxcited States, Vol. 1, E. C. Lim, editor (Academic Press, New York, 1974), p.79.

60. R. Foglizzo and A. Novak, Spec. Lett. 2, 165 (1969). 
This report was done with support from the United States Energy Research and Development Administration. Any conclusions or opinions expressed in this report represent solely those of the author(s) and not necessarily those of The Regents of the University of California, the Lawrence Berkeley Laboratory or the United States Energy Research and Development Administration. 